\title{
Erythropoietin protects against rhabdomyolysis- induced acute kidney injury by modulating macrophage polarization
}

\author{
Shuo Wang ${ }^{1,2,5}$, Chao Zhang ${ }^{1,2,5}$, Jiawei $\mathrm{Li}^{1,2}$, Sidikejiang Niyazi ${ }^{1,2}$, Long Zheng ${ }^{1,2}$, Ming $\mathrm{Xu}^{1,2}$, Ruiming Rong ${ }^{1,2,3}$, Cheng Yang ${ }^{*, 1,2}$ and \\ Tongyu Zhu ${ }^{\star, 1,2,4}$
}

Erythropoietin (EPO) is a well-known hormone that is clinically used for the treatment of anemia. Very recently, an increasing body of evidence showed that EPO could still regulate bioactivities of macrophages. However, the details about the immunomodulatory effect of EPO on macrophages are not fully delineated, particularly in the setting of renal damages. Therefore, in the present study, we determined whether EPO could exert an impact on the dynamics of macrophages in a well-established model of rhabdomyolysis-induced acute kidney injury and explored the potential mechanisms. EPO was found to ameliorate kidney injuries by reducing macrophages recruitment and promoting phenotype switch toward M2 macrophages in vivo. It was also confirmed that EPO could directly suppress pro-inflammatory responses of M1 macrophages and promote M2 marker expression in vitro. Data indicated the possible involvement of Jak2/STAT3/STAT6 pathway in the augmentation of EPO on M2 polarization. These results improved the understanding of the immunoregulatory capacity of EPO on macrophages, which might optimize the therapeutic modalities of EPO.

Cell Death and Disease (2017) 8, e2725; doi:10.1038/cddis.2017.104; published online 6 April 2017

Rhabdomyolysis, as the name indicates, is the disruption of striped (skeletal) muscle followed by massive leakage of electrolytes, myoglobin, and other intracellular proteins into the circulation. ${ }^{1}$ As a severer clinical condition, rhabdomyolysis can be caused by a broad range of physical or chemical damages such as trauma, physical exertion, infections, drugs and toxins, etc. ${ }^{2}$ Acute kidney injury (AKI) is a life-threatening complication of severe rhabdomyolysis and their association was first described during the World War II. ${ }^{3}$ Rhabdomyolysis is recognized now as one of the leading causes of $\mathrm{AKI}$ and rhabdomyolysis-induced $\mathrm{AKI}$ (RIAKI) accounts for $\sim 10 \%$ of all AKI cases. ${ }^{4,5}$ Current treatment for RIAKI relies on supportive therapies and the mortality remains considerably high. ${ }^{6-9}$

A well-established animal model of rhabdomyolysis is induced by intramuscular injection of glycerol and widely used for the exploration of pathogenesis of RIAKI. ${ }^{10}$ The experimental data demonstrated that the mechanisms involved in the pathogenesis of RIAKI included direct injuries on tubular cells by iron release and oxidative stress from myoglobin, as well as indirect damages of subsequent renal vasoconstriction and tubular hypoxia. ${ }^{11-16}$ Interestingly, recent studies shed light into the indispensable role of inflammation and immune cells during the process of RIAKI. ${ }^{17-23}$ Particularly, the dynamics and polarization of macrophages, to a great extent, contributed to the development and repair of renal damages. ${ }^{19-21}$ These studies suggest the macrophage as a novel target to develop effective therapeutics for RIAKI.
Erythropoietin (EPO) is a well-known hormone with classic hematopoietic functions and clinically used for the treatment of anemia. During the past decade, great interest has been paid to the pleiotropic biologic effects of EPO including antiapoptosis, anti-inflammation, neurogenesis and angiogenesis, as well as their consequent tissue protection. ${ }^{24,25}$ Recently, an increasing body of evidence showed that EPO could still modulate bioactivities of immune cells. ${ }^{26-30}$ Specifically for macrophages, EPO has been demonstrated to suppress pro-inflammatory cytokines and augment the phagocytic capacity. ${ }^{26,29,30}$ However, the details about the immunoregulatory effect of EPO remain elusive, particularly in the settings of various diseases. Therefore, in the present study, we explored whether EPO could exert an impact on the dynamics of macrophages in response to RIAKI and the potential mechanisms. Our results provided a novel recognition of EPO for macrophage regulation during AKI, which may help optimize the therapeutic strategies for EPO and develop effective EPO derivatives.

\footnotetext{
Results

EPO protected against rhabdomyolysis-induced kidney injury in mice. We initially confirmed the protection of EPO against rhabdomyolysis-associated renal injury in C57/BL6J mice. The protocols for rhabdomyolysis induction in vivo are summarized in Figure 1. EPO (rhEPO, 3SBIO, Inc.,
}

\footnotetext{
${ }^{1}$ Department of Urology, Zhongshan Hospital, Fudan University, Shanghai, China; ${ }^{2}$ Shanghai Key Laboratory of Organ Transplantation, Shanghai, China; ${ }^{3}$ Department of Transfusion, Zhongshan Hospital, Fudan University, Shanghai, China and ${ }^{4}$ Shanghai Public Health Clinical Center, Fudan University, Shanghai, China

*Corresponding author: C Yang or T Zhu, Department of Urology, Zhongshan Hospital, Fudan University or Shanghai Key Laboratory of Organ Transplantation, 180 Fenglin Road, Shanghai 200032, China. Tel: +86-21-64041990; Fax: +86 21 64037269; E-mail: esuperyc@163.com or tyzhu@fudan.edu.cn

${ }^{5}$ These authors contributed equally to this work.

Received 05.12.16; revised 02.2.17; accepted 15.2.17; Edited by Y Shi
} 
Shengyang, China) was administered at a dose of $500 \mathrm{lU} / \mathrm{kg}$ intraperitoneally $30 \mathrm{~min}$ and $24 \mathrm{~h}$ after glycerol (SigmaAldrich (Shanghai) Trading, Co., Ltd) injection. Glycerol injection caused obvious rises in serum creatinine and blood urea nitrogen (BUN) compared with saline-injected control mice. In contrast, treatment of EPO significantly improved kidney function (Figures $2 a$ and $b$ ). In addition, renal mRNA expressions of kidney injury molecule-1 (KIM-1) and neutrophil gelatinase-associated lipocalin (NGAL) markedly increased after rhabdomyolysis induction while EPO administration significantly reduced $\mathrm{KIM}-1$ as well as NGAL mRNA expression, consistent with renal function results (Figures $2 \mathrm{c}$ and d).

As shown in Figure 2e, intramuscular injection with $50 \%$ glycerol resulted in severe damage of kidney structures especially at the corticomedullary junction, characterized by tubular necrosis, tubular dilation, and cast formation. ATN score was used to grade the histologic alterations based on hematoxylin and eosin (H\&E) staining. EPO obviously improved tubular injury and lowered the ATN score (Figure 2e) in contrast to those of phosphate-buffered saline (PBS)-treated mice. More precisely, we performed periodic acid-Schiff (PAS) staining to clearly exhibit intratubular cast and brush border loss. Although both intratubular cast and brush border stain strongly with PAS, their structures can be easily distinguished due to the paucity of nuclei for intratubular casts (Figure 2f). The extent of casts and preservation of brush borders were further semiquantitatively analyzed. In accordance to the morphologic changes in H\&E-stained slides, treatment of EPO preserved more brush borders and diminished intraluminal casts as shown in Figure $2 f$.

To evaluate apoptosis in kidney tissues in situ during RIAKI, TdT mediated dUTP nick end labeling (TUNEL) staining was conducted. Subjection to glycerol caused a significant rise in apoptotic cells at the corticomedullary junction. Consistent with kidney function and morphology, administration of EPO suppressed apoptosis markedly (Figure $2 \mathrm{~g}$ ).

EPO ameliorated RIAKI-associated renal inflammation. Renal mRNA expressions of cytokines and chemokines were detected by real-time quantitative PCR (RT-qPCR) to evaluate the inflammatory state of kidneys. Levels of proinflammatory cytokines including TNF- $\alpha, \mathrm{IL}-1 \beta, \mathrm{IL}-6, \mathrm{IL}-12 \mathrm{~b}$, IFN- $\gamma$ and chemokines including $\mathrm{C}-\mathrm{C}$ motif chemokine ligand 2 (CCL2) and 7 (CCL7) significantly increased after rhabdomyolysis induction compared with PBS-treated mice, and these upregulations were partially reversed by treatment of EPO (Figures 3a-f). Similar results were also obtained in the determination of TNF- $\alpha$, IL- $1 \beta$ and IL- 6 levels in murine serum (Figures $3 \mathrm{j}-\mathrm{I}$ ). These results were consistent with functional and histologic data and indicated an immunomodulatory role of EPO.

EPO modulated macrophage infiltration and polarization in kidneys during RIAKI. Considering macrophage plays a crucial role in the development of RIAKI, we further explored the infiltration and phenotypes of macrophages. By flow cytometry, pan-leukocyte marker CD45 and macrophage marker CD11b, F4/80 were used to distinguish macrophages in kidney single cell suspension. Gating strategy was

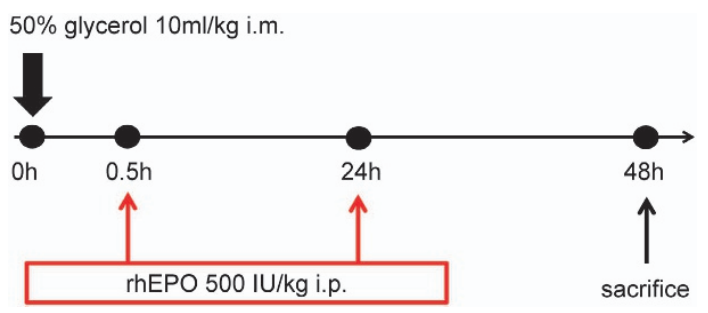

Figure 1 Experimental protocol for the induction of rhabdomyolysis-induced acute kidney injury in mice. 50\% glycerol was administered intramuscularly into the hind limbs of mice at a dose of $10 \mathrm{ml} / \mathrm{kg}$. Saline-injected mice were used as controls. To determine the protective effect of EPO, $500 \mathrm{IU} / \mathrm{kg}$ body weight of EPO or phosphate-buffered saline (PBS) was administered intraperitoneally at the indicated time point post-AKI. Forty eight hours after glycerol injection, mice were killed for blood and kidney samples

exemplified in Supplementary Figure S1. Cells with both expressions of $\mathrm{CD} 11 \mathrm{~b}$ and $\mathrm{F} 4 / 80$ were recognized as macrophages and two populations could be distinguished: $\mathrm{CD}_{11 b^{\text {high }}} \mathrm{F} 4 / 80^{\text {low }}(\mathrm{R} 1)$, and $\mathrm{CD} 11 \mathrm{~b}^{+} \mathrm{F} 4 / 80^{\text {high }}(\mathrm{R} 2)$. The percentage of $\mathrm{CD} 11 \mathrm{~b}^{+} \mathrm{F} 4 / 80^{+}$cells among whole renal cells was calculated and served as a parameter for the infiltration of macrophages. As shown in Figure $4 \mathrm{a}$, mice subjected to glycerol demonstrated a dramatic recruitment of macrophages toward kidneys while treatment of EPO alleviated such infiltration significantly. The dynamics of subpopulations $\mathrm{R} 1$ and $\mathrm{R} 2$ was also analyzed. R2 cells are recognized as the resident macrophages within kidneys and presents with M2like phenotype. Accordingly, our results confirmed the predominance of R2 in kidneys from control group, and demonstrated a dramatic increase in $\mathrm{R} 1$ population after rhabdomyolysis induction. However, the ratio of R2/R1 at 2 days after glycerol injection was partial reversed by EPO administration. More accurately, anti-CD206 antibody was used to determine the M2 phenotype. The data showed that the proportion of M2 macrophages increased during RIAKI and was further upregulated by EPO. Additionally, immunohistochemical staining for F4/80 was performed and confirmed the flow cytometry data that macrophages infiltration was diminished by EPO (Figure 4b). Importantly, mRNA levels of M2 markers including arginase-1, Ym-1, Fizz-1 and CD206 were examined by RT-qPCR and suggested EPO could enhance polarization toward M2 macrophages (Figure 4c).

EPO inhibited apoptosis and reduced chemokine production of tubular epithelial cells in response to myoglobin. Cell death serves as an important initiator of recruitment of immune cells and subsequent inflammatory responses. The anti-apoptotic effect of EPO has been widely reported and was recognized as a key contributor to its renoprotection. However, the current prevailing theory believes that necroptosis, a novel identified cell death, plays a crucial role in the pathogenesis of $\mathrm{AKI}$, even outweighs apoptosis. ${ }^{31-33}$ Therefore, it is indeed necessary to explore whether EPO could modulate necroptosis in the setting of RIAKI. Therefore, we subjected human renal proximal tubular epithelial cell line (HK-2) to myoglobin to mimic rhabdomyolysis-associated kidney injuries in vitro. Graded 


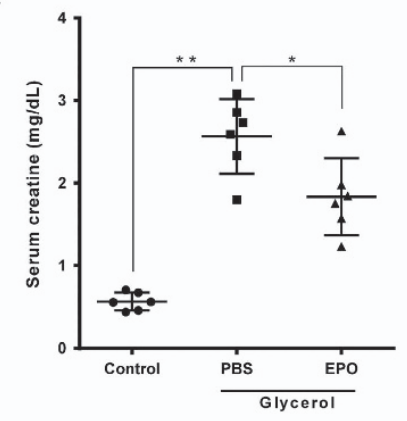

b

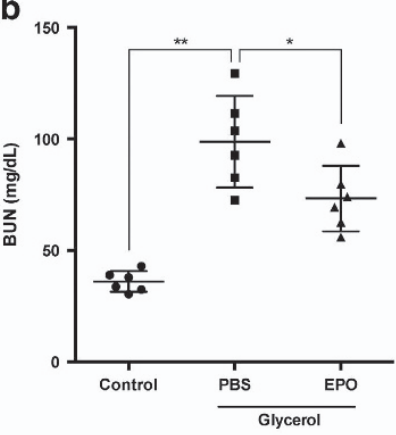

d

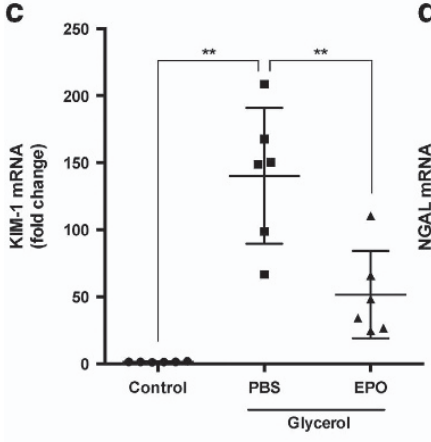

d

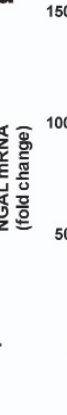

e
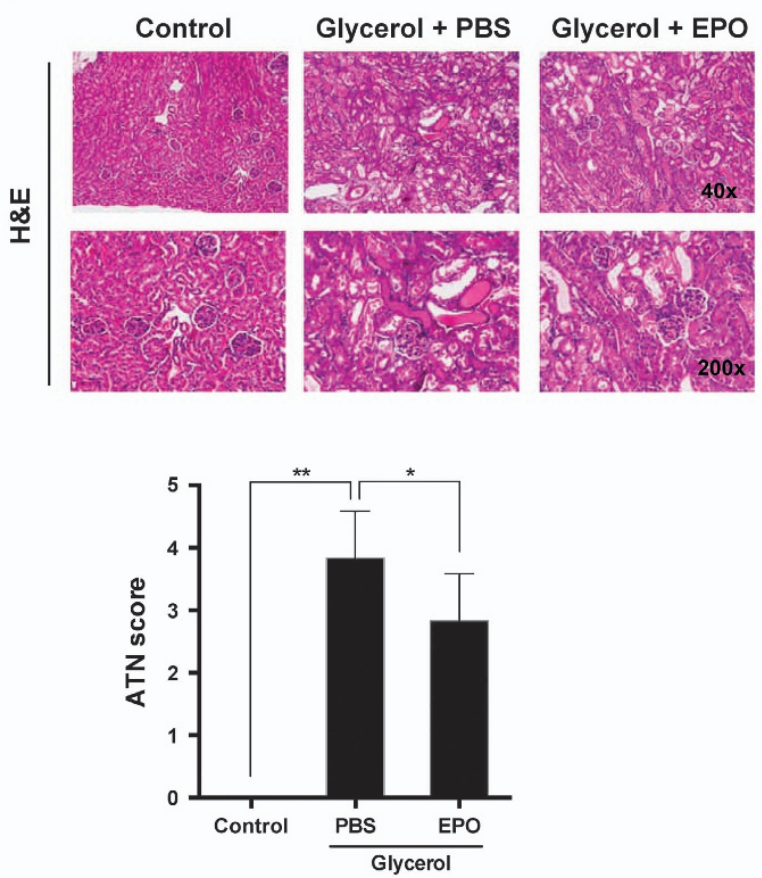

f
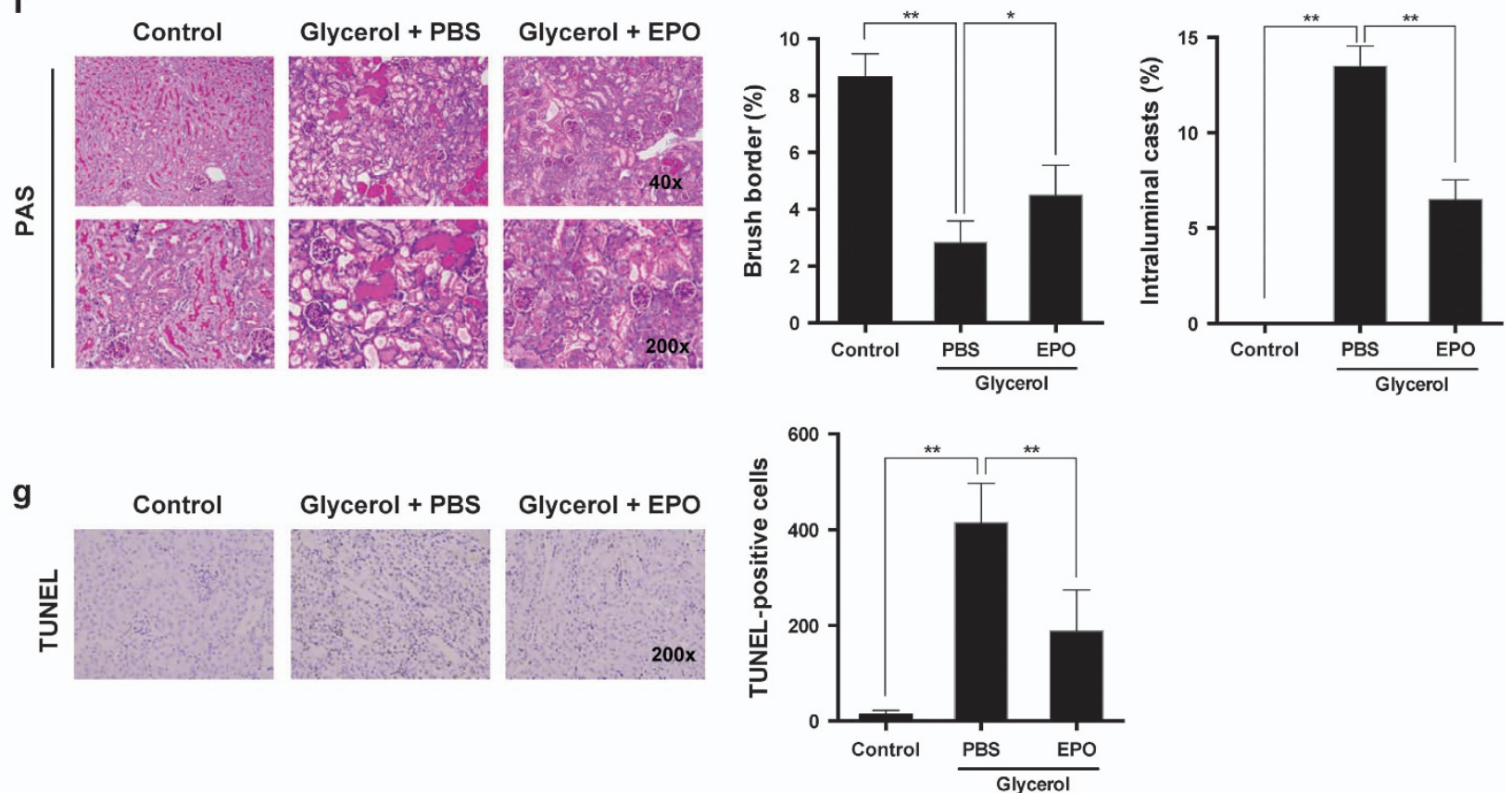

Figure 2 EPO improved renal damages in RIKAI. Mice subjected to different treatments were killed at the indicated time point. Serum levels of (a) creatinine and (b) blood urea nitrogen (BUN) were detected. Renal mRNA levels of (c) KIM-1 and (d) NGAL were analyzed by qPCR. (e) Representative images of H\&E staining are exhibited and ATN scores were calculated. (f) Representative images of PAS staining and quantification of brush borders as well as intraluminal casts were showed. (g) Representative photographs of TUNEL staining ( $\times 200)$. Quantitative analysis of TUNEL-positive cells was performed. Data are expressed as mean \pm S.D. $(n=6)$. ${ }^{\star} P<0.05,{ }^{* \star} P<0.01$

concentrations of myoglobin $(1,5$, and $10 \mathrm{mg} / \mathrm{ml})$ were tested, and only at $10 \mathrm{mg} / \mathrm{ml}$ could myoglobin induce obvious cell death (data not shown). Flow cytometry analysis was conducted to detect different types of cell death based on Annexin V/PI staining: Annexin $\mathrm{V}^{-} / \mathrm{PI}^{-}$are live cells; Annexin $\mathrm{V}^{+} / \mathrm{PI}^{-}$represent early apoptosis; Annexin $\mathrm{V}^{-} / \mathrm{PI}^{+}$reflect necroptosis; Annexin $\mathrm{V}^{+} / \mathrm{PI}^{+}$include both late apoptosis and necroptosis. In accordance with previous studies, ${ }^{21,34}$ exposure to myoglobin caused a significant decrease in cell viability in contrast with vehicle-treated HK-2 cells. Moreover, addition of Nec-1, a widely used inhibitor for necroptosis, obviously diminished $\mathrm{Pl}^{+}$cell death, indicating myoglobin 


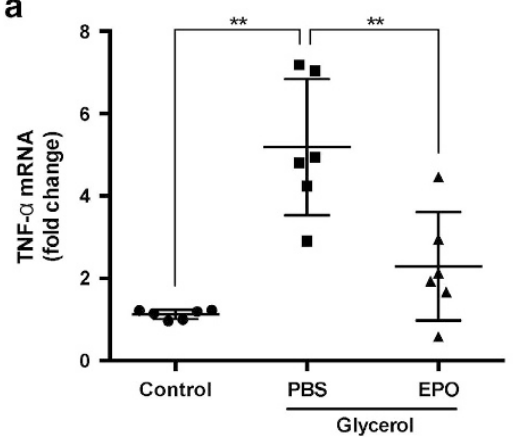

d

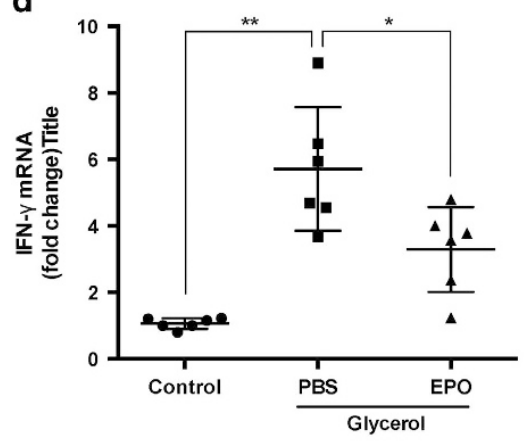

g

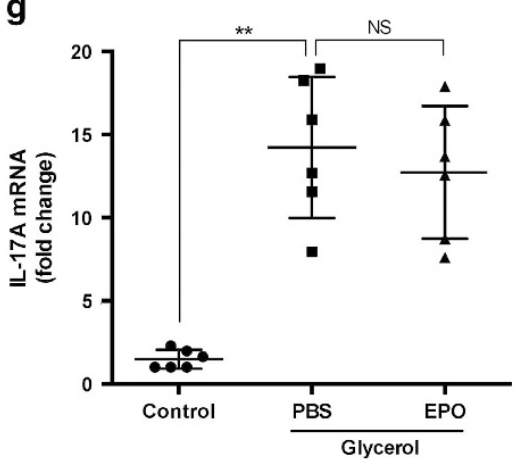

j

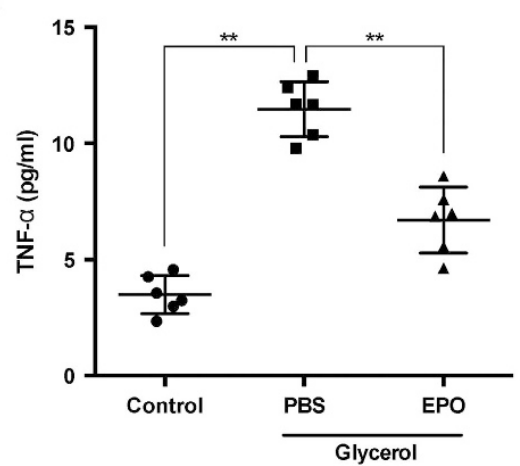

b

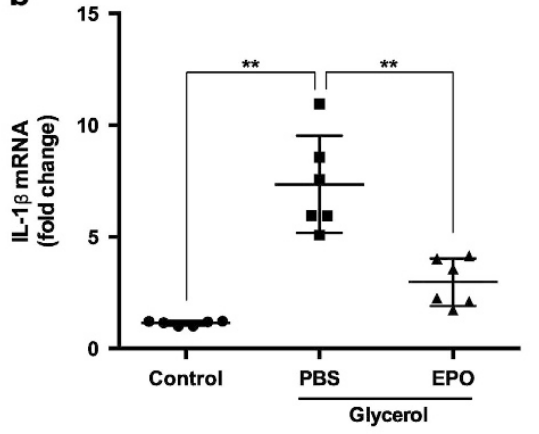

e

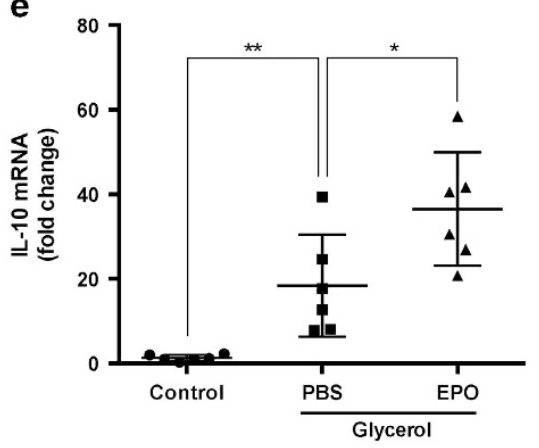

h

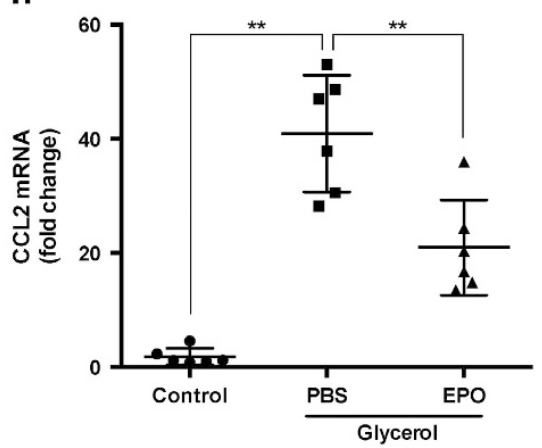

k

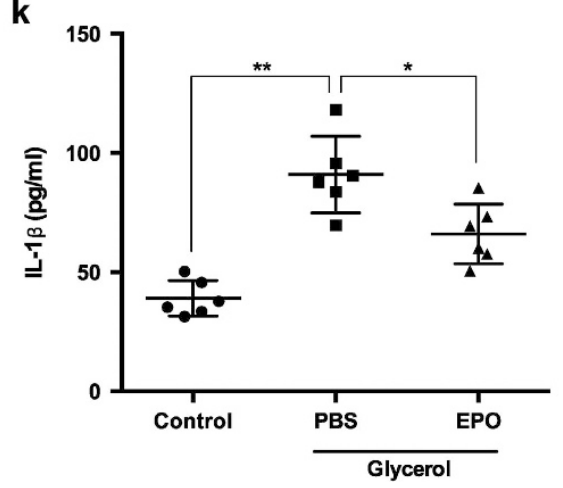

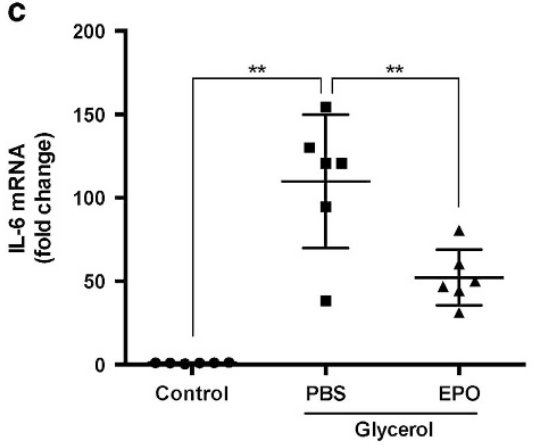

f

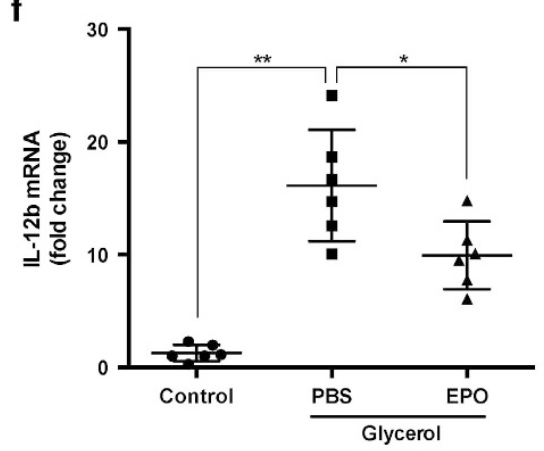

i

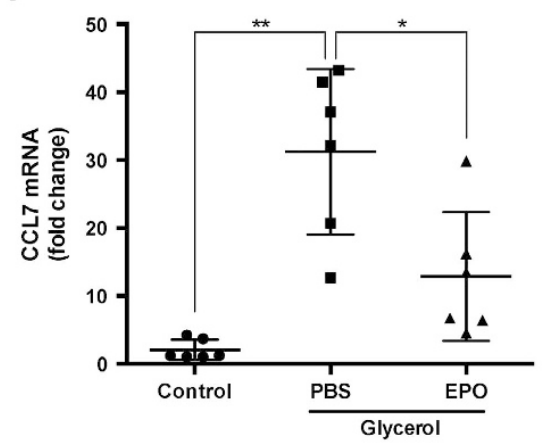

I

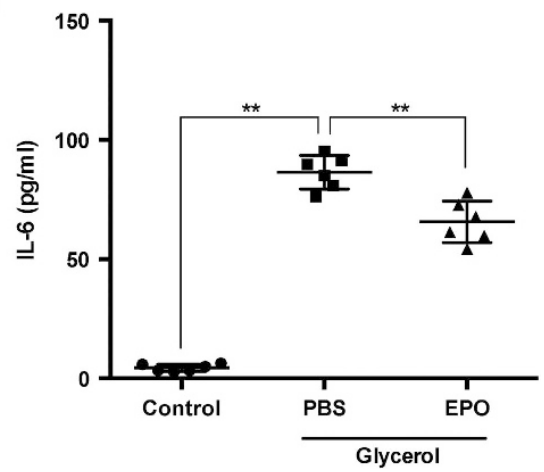

Figure 3 EPO alleviated inflammatory responses in RIAKI. Renal mRNA levels of (a) TNF- $\alpha$, (b) IL-1 $\beta$, (c) IL-6, (d) IFN- $\gamma$, (e) IL-10, (f) IL-12b, (g) IL-17 A, (h) CCL2, and (i) CCL7 were analyzed by qPCR. Serum levels of (j) TNF- $\alpha$, (k) IL-1 $\beta$, and (I) IL-6 were determined. Data are expressed as mean \pm SD $(n=6)$. ${ }^{\star} P<0.05$, ${ }^{\star \star} P<0.01$

Figure 4 EPO diminished macrophage infiltration and regulated macrophage polarization within kidneys during RIAKI. (a) Intact kidneys were decapsulated, diced, and digested for single cell suspensions. Renal single cells were then incubated with anti-mouse CD45, CD11b, F4/80 and CD206 antibodies and subsequently analyzed by flow cytometry. Macrophages can be divided into two subgroups: $\mathrm{CD} 11 \mathrm{~b}^{\text {high }} \mathrm{F} 4 / 80^{\text {low }}(\mathrm{R} 1)$, and $\mathrm{CD} 11 \mathrm{~b}^{+} \mathrm{F} 4 / 8 \mathrm{~h}^{\text {high }}$ (R2). Quantitative analysis of macrophages $\left(\mathrm{CD} 11 \mathrm{~b}^{+} \mathrm{F} 4 / 80^{+}\right)$percentage, ration of R2/R1, and M2 (CD206 ${ }^{+}$) percentage was performed. (b) Representative photographs of anti-F4/80 staining at $\times 200$ magnification. Arrows suggested macrophages located within renal interstitium. (c) Renal mRNA levels of arginase-1, Ym-1, Fizz-1 and CD206 were detected by qPCR. Data are expressed as mean \pm S.D. $(n=6)$. ${ }^{*} P<0.05$, ${ }^{* \star} P<0.01$ 
could induce necroptosis as well. The results also demonstrated that treatment of EPO diminished Annexin $\mathrm{V}^{+}$cell death (Figure 5a). Considering Annexin $\mathrm{V}^{+} / \mathrm{PI}^{+}$contained an overlapping cell spectrums of both necroptosis and apoptosis, western blot was further used to delineate the effects of EPO on necroptosis and apoptosis separately. Activated

a Control
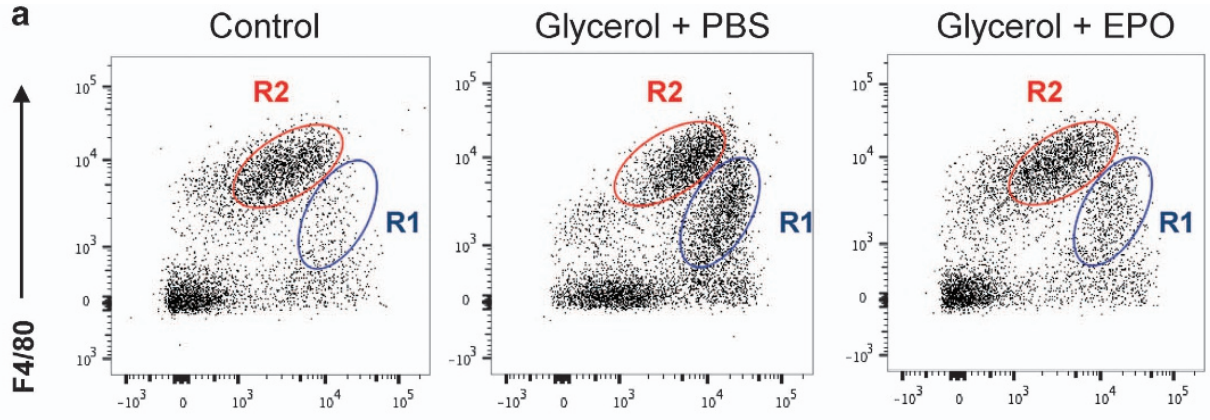

CD11b
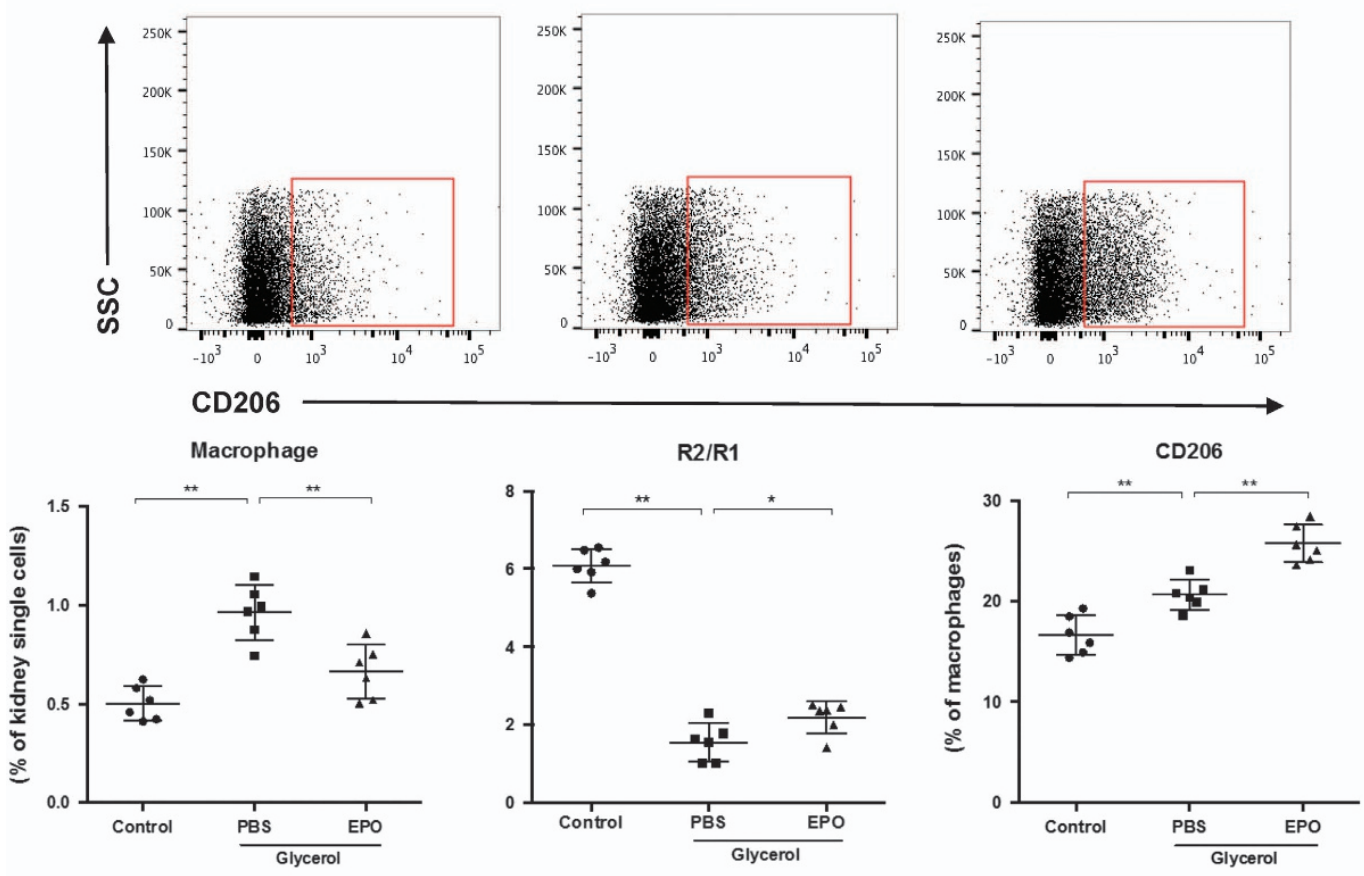

b

Control

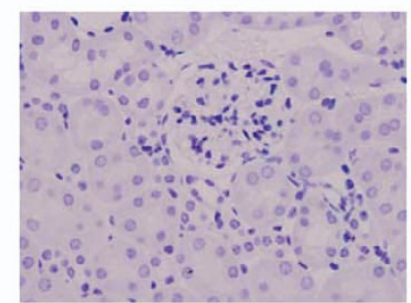

Glycerol + PBS

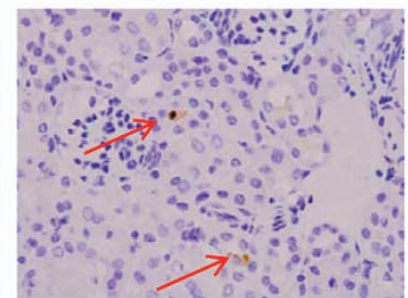

Glycerol + EPO

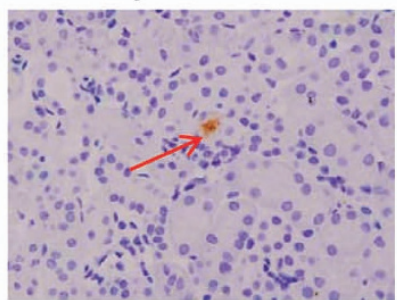

c
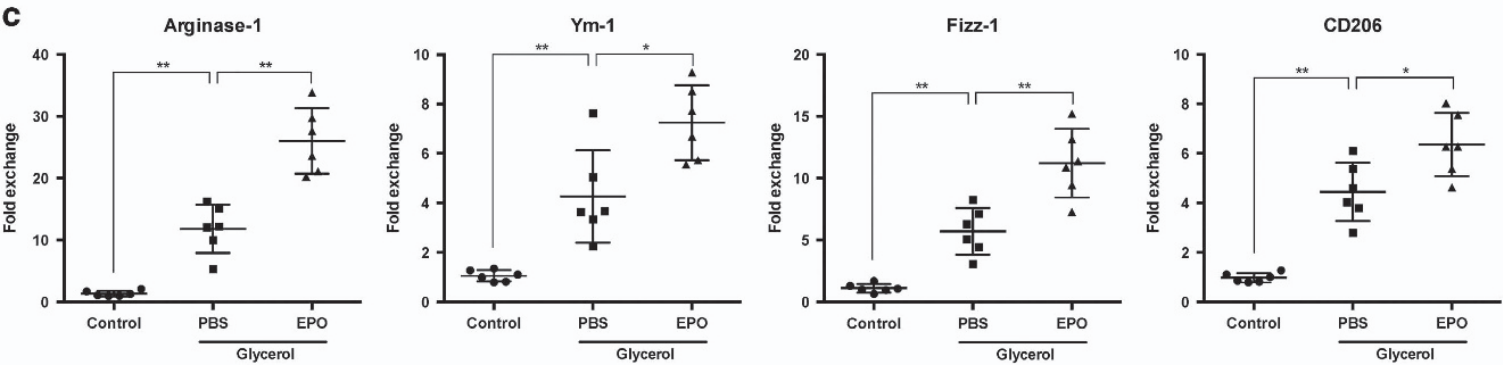
hRIP3 directly phosphorylates hMLKL at the Thr357 and Ser358 sites, phosphorylated hMLKL has been recognized as useful biomarkers for the characterization of necroptosis in vitro. ${ }^{35}$ As shown in Figure 5b, EPO decreased the level of cleaved caspase-3 but had no impact on phosphorylated MLKL (Ser358), suggesting EPO could only inhibit apoptosis

a
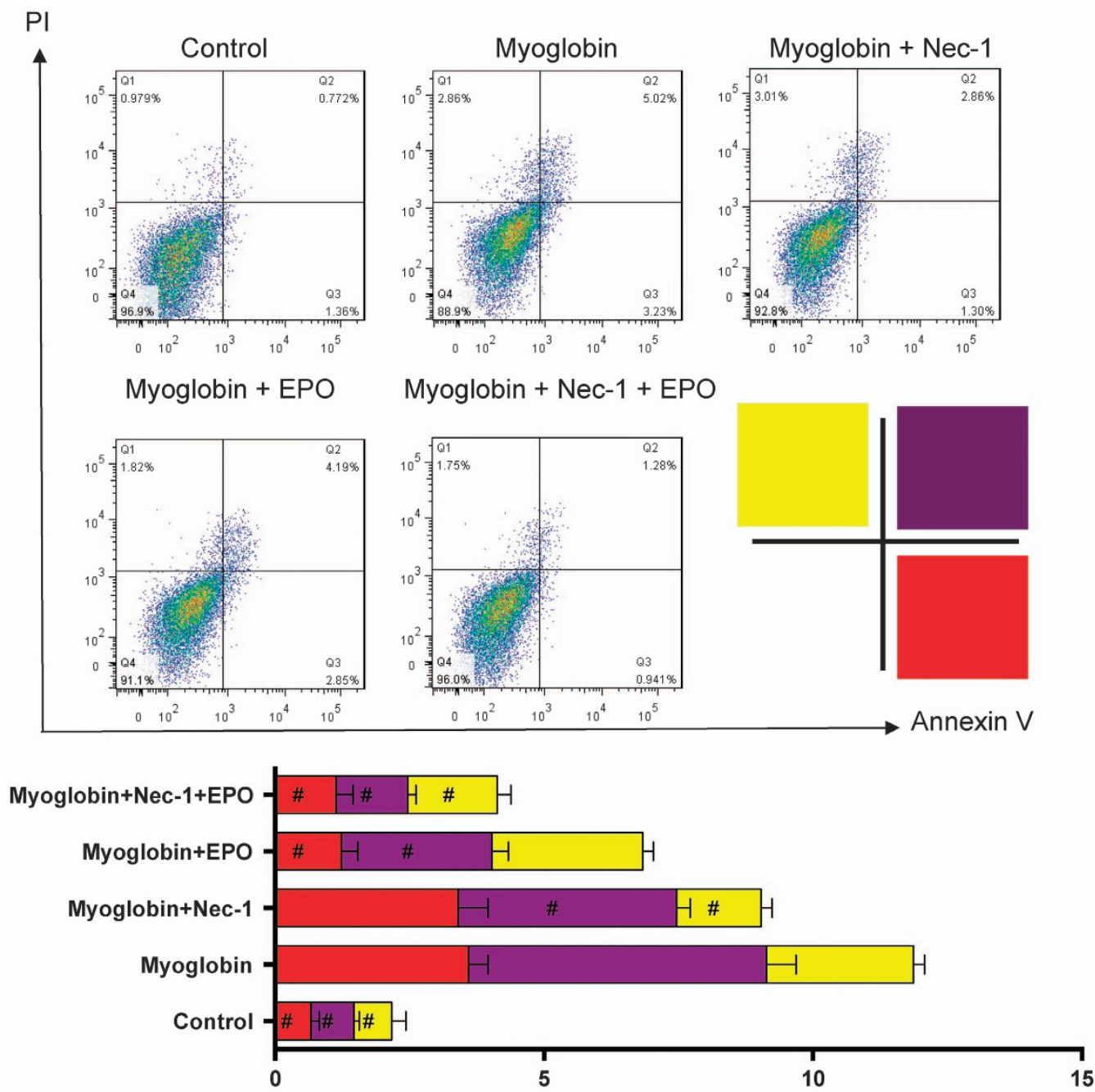

(\%)

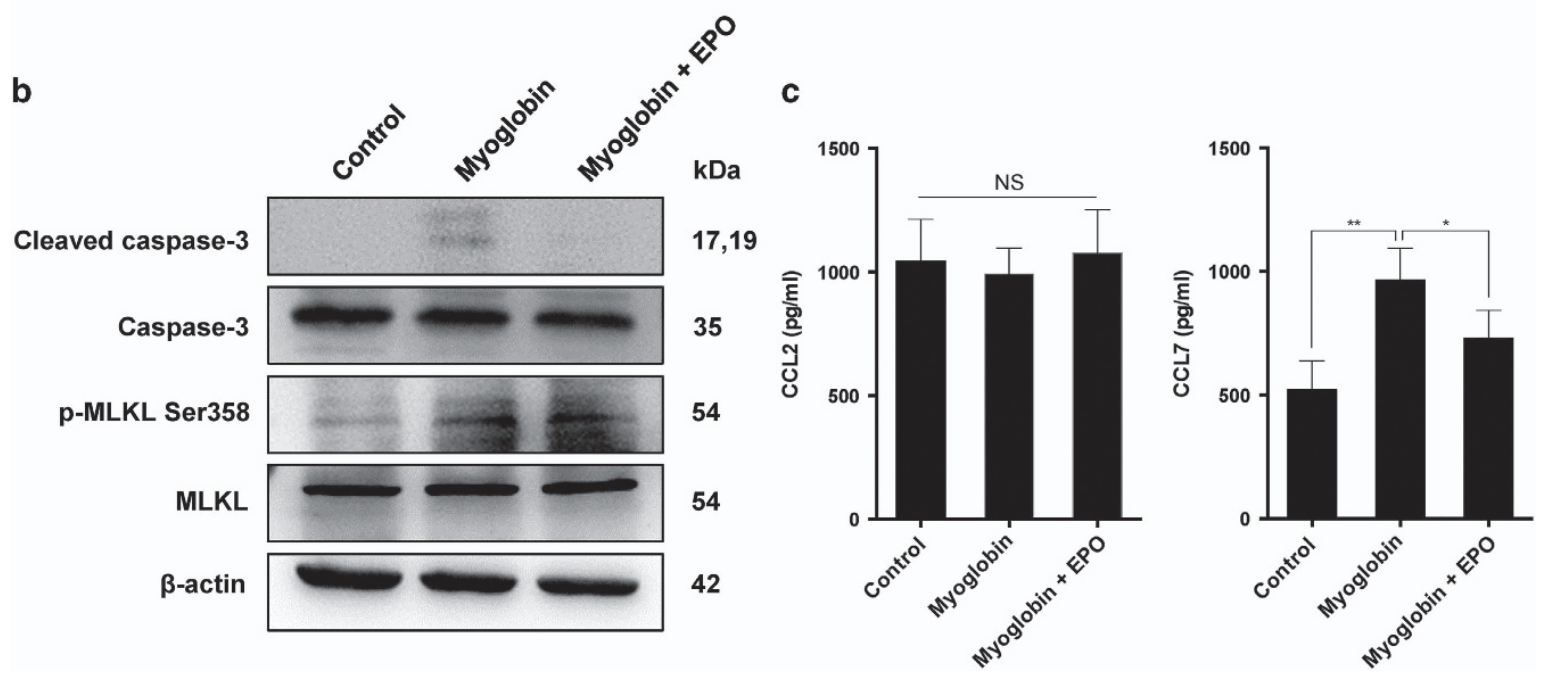


but not necroptosis that is more pro-inflammatory. Considering CCL2 and CCL7 play key roles in the infiltration of macrophages, ${ }^{36}$ we further determined the secretion of CCL2 and CCL7 in HK-2 cells in response to myoglobin. Myoglobin caused an increase in the production of CCL7 and treatment of EPO partially reversed the CCL7 level (Figure 5c). Overall, these data suggested that EPO could suppress the recruitment of macrophages by inhibiting apoptosis and chemokine secretion.

EPO facilitated macrophage polarization toward alternative activation (M2) in vitro. Next, we determined whether EPO was able to regulate macrophage phenotypes directly. As shown in Figure 6, EPO reduced the concentrations of pro-inflammatory molecules including NO, TNF- $a$, $\mathrm{IL}-1 \beta$, and $\mathrm{IL}-6$ in a dose-dependent manner in both RAW264.7 macrophage cell line and primary bone marrowderived macrophage (BMDM), which were stimulated by a combination of LPS and IFN- $\gamma$. Alternative activation of macrophages (M2) was initiated by addition of IL-4. Importantly, co-treatment of IL-4 and EPO upregulated the mRNA expressions of arginase-1, Ym-1, Fizz-1, and CD206 which were indicators of M2 macrophages (Figure 7). Notably, only in the presence of IL-4 can EPO promote M2 polarization in macrophages, while EPO alone exerted no effect. Taken together, those results indicated that EPO inhibited M1 and augmented M2 phenotypes, and thereby favored an immunoregulatory switching of macrophages.

Jak2/STAT3/STAT6 pathway was involved in the promotion of M2 phenotype by EPO. We subsequently explored the possible mechanism by which EPO promotes M2 polarization. It is well documented that EPO activates a series of signaling pathways including Janus-tyrosine kinase 2 (Jak2)/signal transducer and activator of transcription (STAT), phosphoinositide 3 kinase (PI3K), mitogen-activated protein kinase (MAPK) pathways. In this regard, we evaluated the key participants of these pathways by western blot and found significant upregulations of p-Jak2, p-STAT3, and arginase-1 in EPOtreated macrophages (Figure 8a). Alterations in PI3K and p-p38 expression were also observed but did not reached the statistical significance. Considering EPO could not promote M2 polarization alone, we also determined the expression of p-STAT6, which is a key regulator in the generation of IL-4stimulated M2 macrophage. We further determined the contribution of Jak2/STAT3 to EPO-induced M2 polarization by applying p-Jak2 inhibitor AZD1480 (Selleck) and p-STAT3 inhibitor Stattic (Selleck). As shown in Figure 8b, both inhibitors abolished the EPO-mediated increases in p-STAT6 and arginase-1, indicating that EPO might enhance M2 polarization by upregulating p-STAT6 via Jak2/STAT3 pathway. Proposed pathways was illustrated in Figure 8c.

\section{Discussion}

Despite of an increased insight into the anti-inflammatory effect of EPO in the past decade, the modulation of EPO on immune cells and their exact mechanisms are not fully elucidated. A better understanding of the immunoregulatory capacity of EPO may improve the therapeutic regimens of EPO in the treatment for various diseases. In the present study, we demonstrated that EPO could diminish the rhabdomyolysis-associated kidney injuries by ameliorating the infiltration of macrophages and promoting M2 polarization within kidneys during RIAKI. As shown in Figure 9, EPO exerted its modulation on the dynamics of renal macrophages through pleiotropic mechanisms. Myoglobin caused direct injuries on the tubular epithelial cells and stimulated secretion of chemokine CCL7. In contrast, EPO protected against myoglobin-induced apoptosis and decreased the level of CCL7, thereby inhibiting the recruitment of monocytes/macrophages. In addition, EPO suppressed the pro-inflammatory responses of activated macrophages. More importantly, our data showed that EPO were able to augment the polarization of macrophages toward M2 phenotype in the presence of IL-4. By these mechanisms, EPO significantly altered the paradigm of macrophages in the microenvironment of kidneys and thereby reduced M1-related tissue damages and enhanced the reparative effects of $M 2$. Our research provided a novel recognition of EPO on macrophage subtype switch specifically in the setting of AKI.

An increasing body of studies has shed light into the effects of EPO on macrophage bioactivities. Several studies have proved that EPO could inhibit pro-inflammatory activation of macrophages. ${ }^{26,37,38}$ In accordance with our results, EPO has also been shown to promote macrophage-mediated $\mathrm{T}$ cell suppression $^{39}$ and induce the expansion of M2-like macrophages in white adipose tissue. ${ }^{40}$ These characteristics have also been confirmed in nonerythropoietic EPO derivative ARA290. ${ }^{41-44}$ However, another study obtained the conflicting results that EPO enhanced the pro-inflammatory activity of macrophages. ${ }^{45}$ It remains unknown what results in this discrepancy, which needs further explorations in the future. Interestingly, EPO has been demonstrated to enhance the phagocytic function of macrophages. ${ }^{29,30,37}$ Clearance of damaged tissue can facilitate renal repair and promote immune tolerance. Therefore, the augmentation of phagocytosis of macrophages is likely to be one of the mechanisms for the renoprotective effect of EPO as well. Such hypothesis does not contradict with our study, in which we focused the functional switch of macrophages.

Commonly defined by in vitro experiments, macrophage activation can be distinguished for two major subtypes: classically activated M1 macrophages are pro-inflammatory and exert deleterious effect in sterile tissue injuries; in contrast, alternatively activated M2 macrophages are

Figure 5 EPO inhibited myoglobin-induced apoptosis and chemokine production of tubular epithelial cells in vitro. Human renal proximal tubular epithelial cell line HK-2 was treated with $10 \mathrm{mg} / \mathrm{ml}$ myoglobin for $4 \mathrm{~h}$ in the presence or absence of $50 \mathrm{lU} / \mathrm{ml}$ EPO. (a) To explore a role of necroptosis in myoglobin-induced cell death, a necroptosis inhibitor $\mathrm{Nec}-1(10 \mu \mathrm{M})$ was used. Cells were collected and detected by flow cytometry based on Annexin V/PI staining. (b) Protein was prepared from cultured cells. Expressions of cleaved caspase-3 and phosphorylated MLKL were analyzed by western blotting. (c) Levels of CCL2 and CCL7 in the culture supernatants were determined by ELISA. Data are representative of three independent experiments. Data are expressed as mean \pm S.D. ${ }^{*} P<0.05$, ${ }^{* *} P<0.01$. ${ }^{\#} P<0.05$ compared with myoglobin-treated groups 
RAW 264.7

a

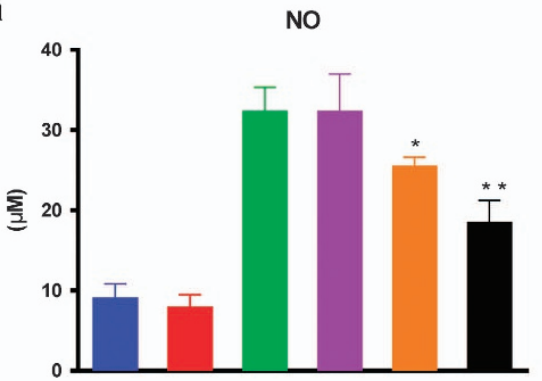

b

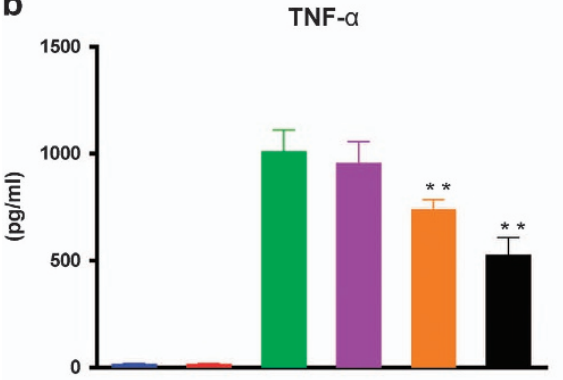

C

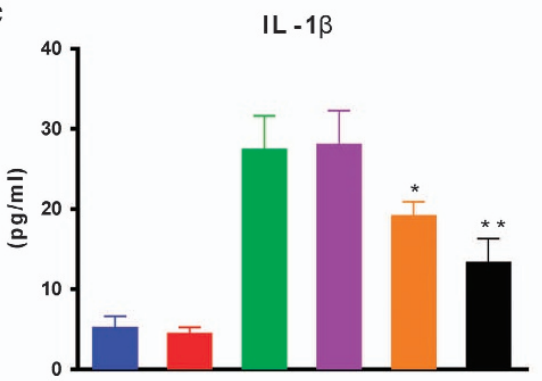

d

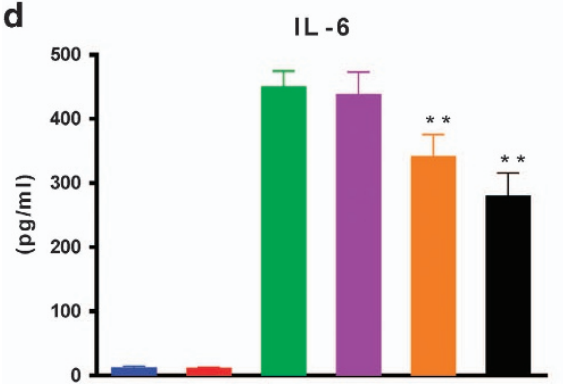

Primary BMDM
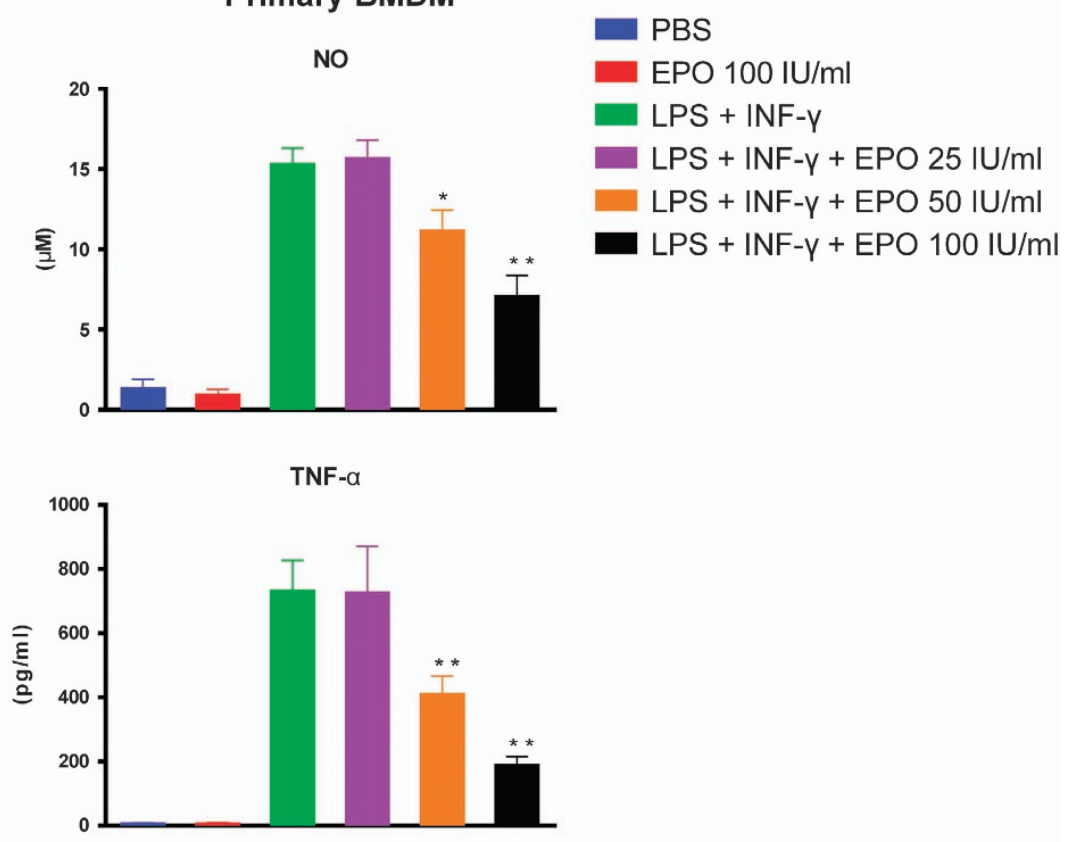

$\mathrm{IL}-1 \beta$

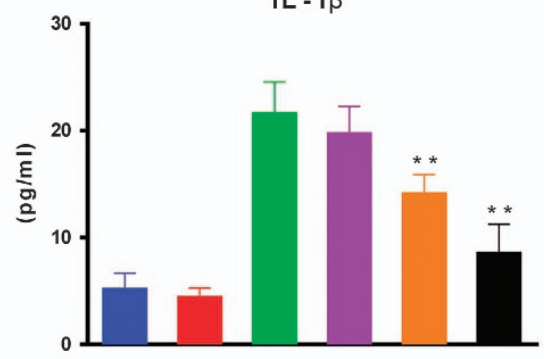

IL -6

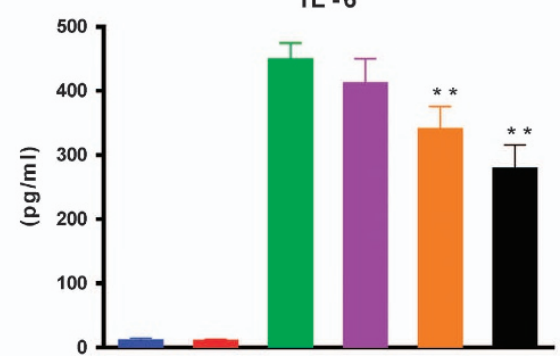

Figure 6 EPO inhibited pro-inflammatory response in M1 macrophages. RAW 264.7 cells and bone marrow-derived macrophages (BMDM) were primed with $1 \mu \mathrm{g} / \mathrm{ml}$ LPS

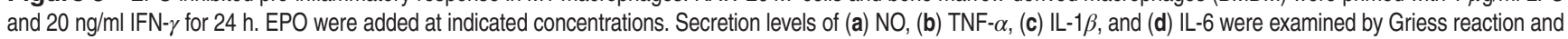
ELISA. Experiments were performed in triplicate. Data are expressed as mean \pm S.D. ${ }^{*} P<0.05,{ }^{\star \star} P<0.01$

characterized by immunoregulatory and tissue repair capabilities. $^{46-48}$ Although indeed simplistic, this essential classification is well accepted and explains the functional plasticity and dynamics of macrophages in response to different insults in vivo. ${ }^{49}$ Typically, renal macrophages are a heterogeneous group with their phenotypes changing under physiological and pathological conditions. ${ }^{50}$ Normally, there are a small number of resident macrophages within kidneys that are of vital importance for maintaining homeostasis of renal microenvironments. ${ }^{51,52}$ Upon outsets of diverse AKI, primary renal damages are inevitably followed by a rapid influx of abundance of monocytes that subsequently differentiate into macrophages. ${ }^{53,54}$ In the early stage of $\mathrm{AKI}$, infiltrated macrophages present as pro-inflammatory $\mathrm{M} 1$ phenotype and consequently amplify tissue damage by secretion of cytokines and induction of apoptosis. Over a period of time, the predominance of macrophages switches to the anti-inflammatory M2 subtype, which facilitate post-AKI repair.

Endogenous factors in the kidney microenvironment regulate the polarization dynamics of macrophages and the underlying mechanisms are under intensively 
RAW 264.7

a

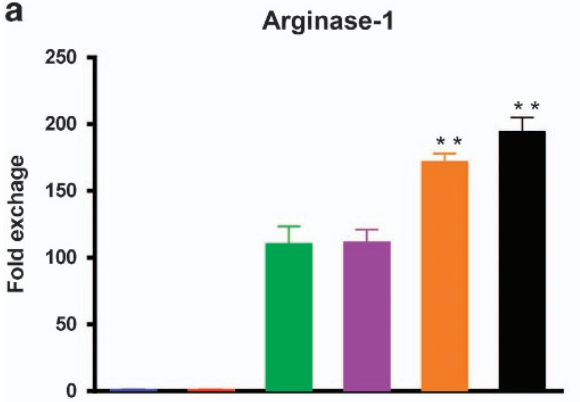

b

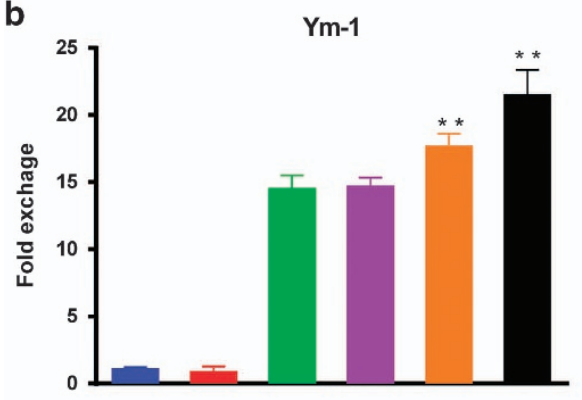

C

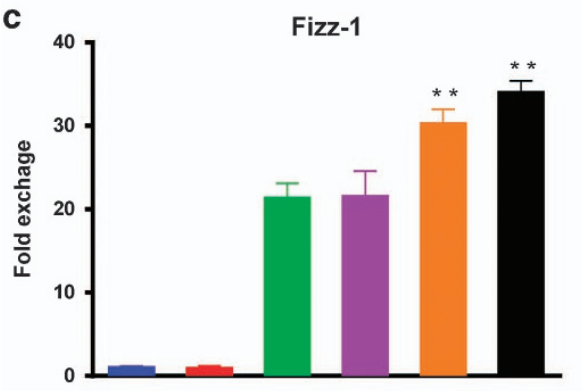

d

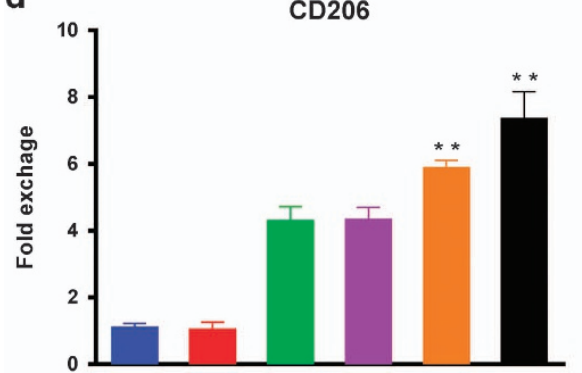

Primary BMDM

PBS

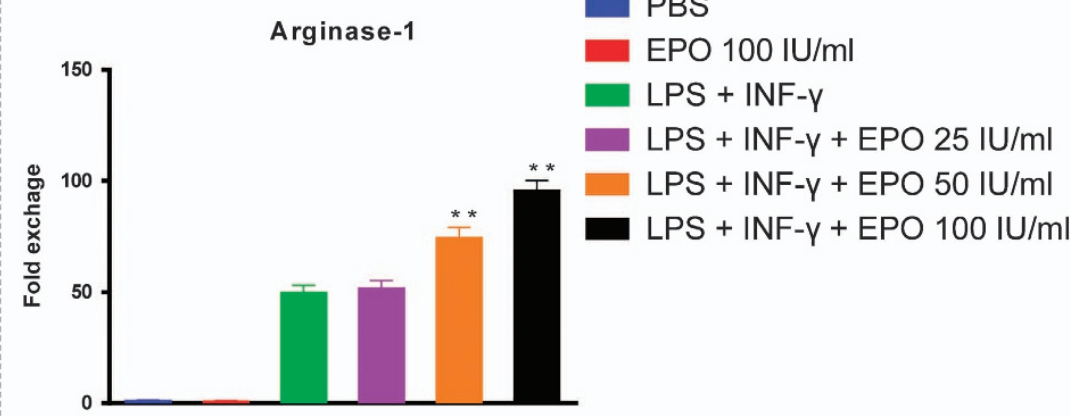

Ym-1

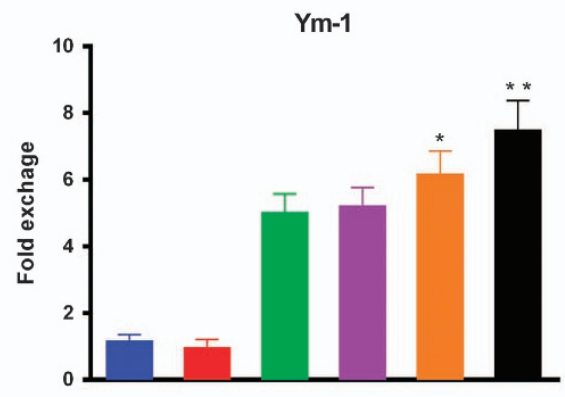

Fizz-1

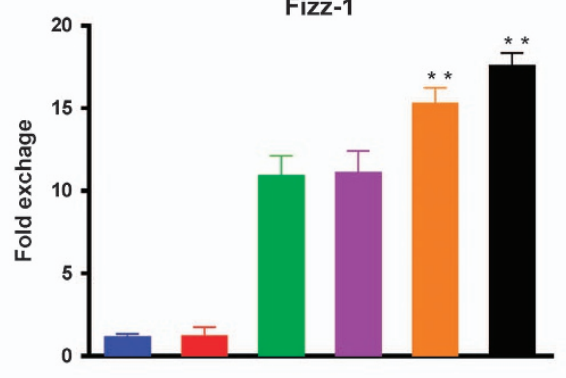

CD206

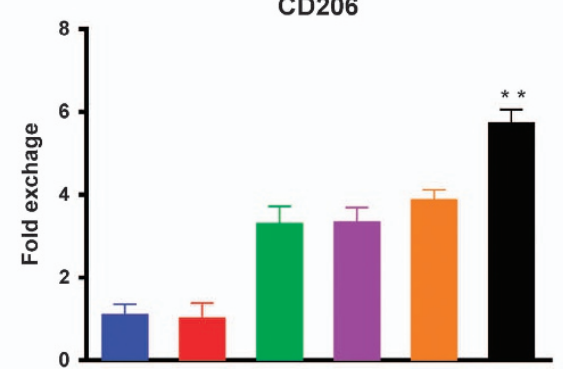

Figure 7 EPO promoted the expression of M2 markers. RAW 264.7 cells and bone marrow-derived macrophages (BMDM) were stimulated with $20 \mathrm{ng} / \mathrm{ml}$ IL-4 for $24 \mathrm{~h}$. EPO were added at indicated concentrations. Cellular mRNA expressions of (a) arginase-1, (b) Ym-1, (c) Fizz-1, and (d) CD206 were detected by qPCR. Experiments were performed in triplicate. Data are expressed as mean \pm S.D. ${ }^{*} P<0.05,{ }^{*} P<0.01$

investigation recently. Retinoic acid, colony-stimulating factors 1 and 2 have been demonstrated to promote the alternative activation of macrophages during AKI. ${ }^{55-59}$ The dynamic equilibrium between $\mathrm{M} 1$ and $\mathrm{M} 2$ macrophages plays a crucial role in the initiation and development of kidney damages. In this regard, it is of great value to explore how to therapeutically manipulate this balance favorably toward M2 polarization. Mesenchymal stem cells, for instance, have been reported to improve RIAKI by activation of M2 macrophages. $^{20}$
Previous research observed that adoptive transfer of M2 macrophages led to an attenuation of renal pathology. ${ }^{60}$ In this study, we showed that EPO could facilitate M2 polarization via Jak2/STAT3/STAT6 pathway that may suggest another mechanism of the tissue protection of EPO against AKI. Of note, the phenotypic evolving of renal macrophages during $\mathrm{AKI}$ - as discussed above - is a complex process, which is orchestrated by diverse factors. In our study, however, we simplified M2 polarization in vitro by using classic IL-4-primed macrophages. Strictly, macrophage-specific epo receptor 
a

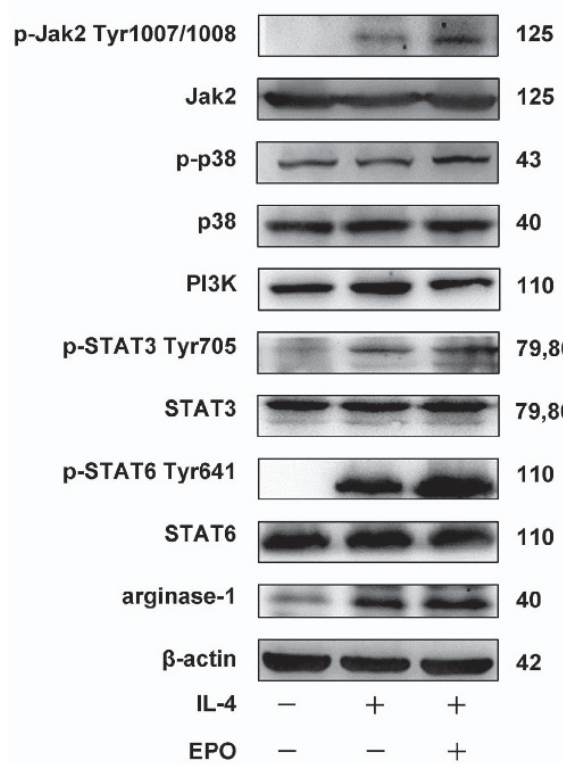

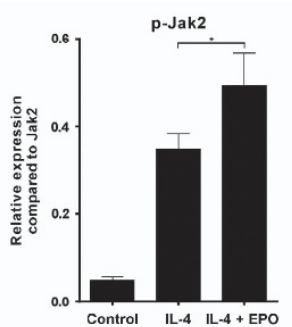
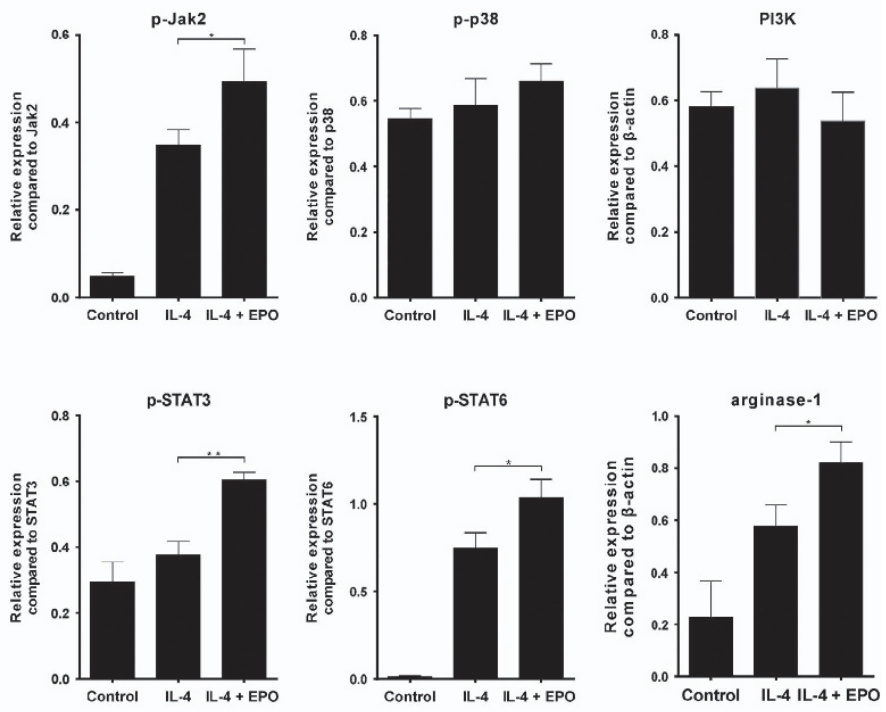

b

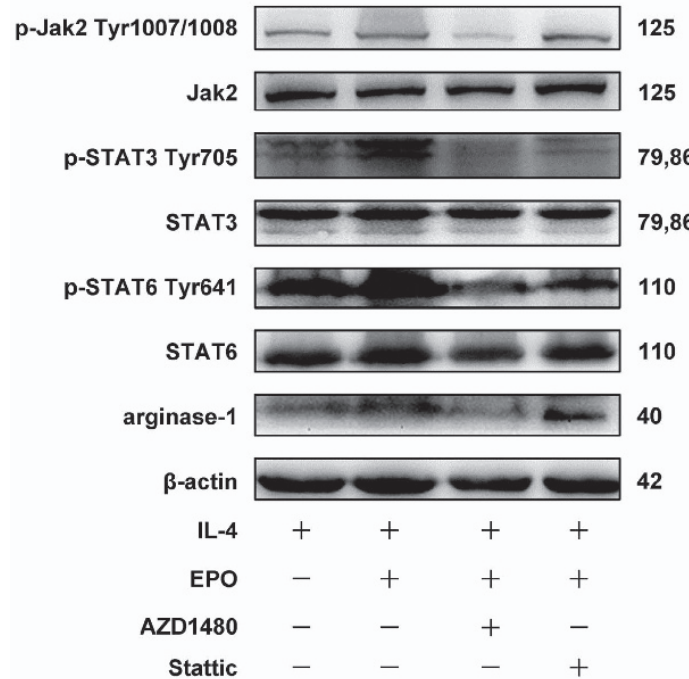

c

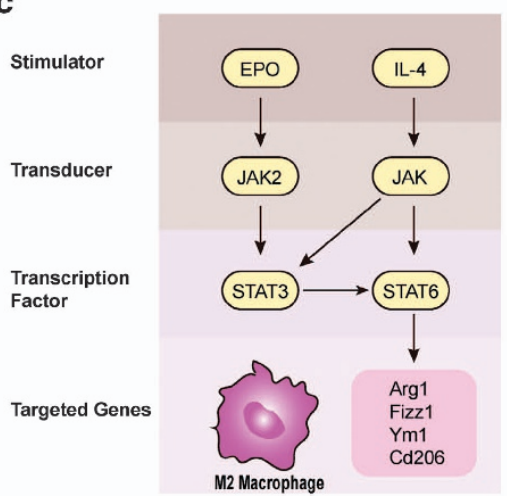

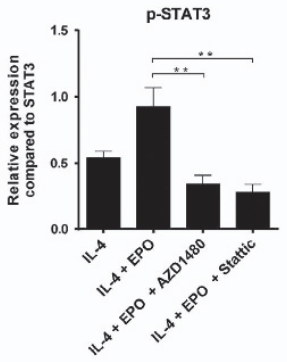
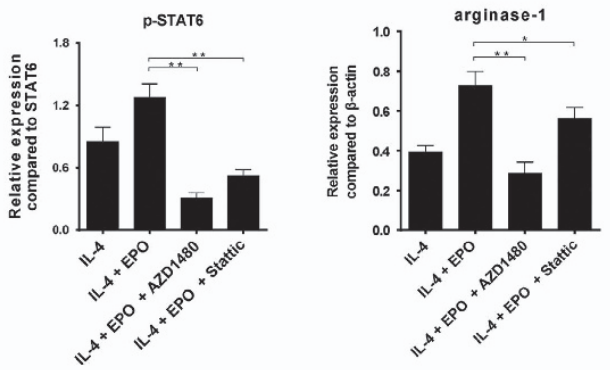

Figure 8 EPO augmented M2 polarization via Jak2/STAT3/STAT6 pathway. (a) RAW 264.7 cells were subjected to $20 \mathrm{ng} / \mathrm{ml}$ IL-4 in the presence or absence of EPO $(50 \mathrm{IU} / \mathrm{ml})$ for $24 \mathrm{~h}$. Protein expressions of related molecules were measured and representative photographs are exhibited. (b) To further confirm the contribution of Jak2/STAT3, RAW 264.7 cells stimulated by IL-4 and EPO were incubated with AZD1480 (an inhibitor of p-Jak2, $5 \mu \mathrm{M}$ ) or Stattic (an inhibitor of p-STAT3, $10 \mu \mathrm{M}$ ). Protein expressions of related molecules were measured and representative images are demonstrated. (c) Proposed pathways by which EPO promotes M2 polarization in the presence of IL-4. Experiments were performed in triplicate. Error bars represent S.D. ${ }^{*} P<0.05,{ }^{* \star} P<0.01$ 


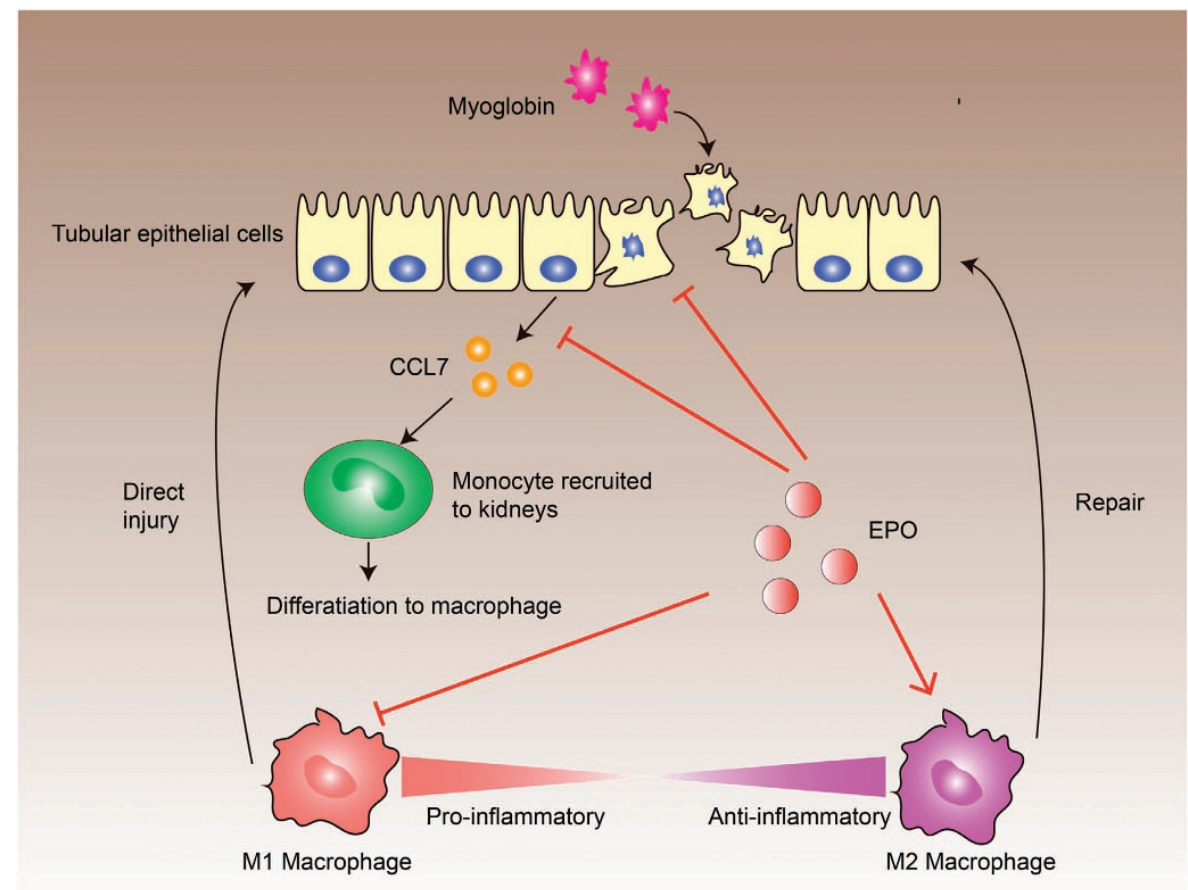

Figure 9 Proposed mechanisms involved in the modulation of macrophage phenotypes by EPO during rhabdomyolysis-induced acute kidney injury. Myoglobin can induce both necroptosis and apoptosis of tubular epithelial cells and initiate the secretion of chemokine CCL7, which recruit monocytes and serve as key initiator of the subsequent inflammation. EPO could only inhibit apoptosis, but not necroptosis that are more destructive and pro-inflammatory. On the other hand, EPO decreases the level of secreted chemokine CCL7, thus consequently suppresses the migration of monocytes into kidneys. Moreover, EPO can directly inhibit the activation of M1 subtypes and promotes polarization toward M2 phenotypes. As a result, the M1/M2 ratio during rhabdomyolysis-induced acute kidney injury is favorably regulated toward an anti-inflammatory phenotype and thereby ameliorates the renal damages

knockout mice model are needed to further identify the effect of EPO on macrophages in vivo and in vitro, which is warranted in the future study.

Moreover, a number of reports indicated that M2 macrophages within kidneys could promote renal fibrosis in addition to their reparative effects on tissue damages. ${ }^{61,62}$ In this regard, although there is no solid evidence suggesting the side effect of EPO on fibrosis in the treatment for AKI, the possible risk should take into account in the following researches.

In conclusion, the present study demonstrated that EPO could reduce macrophages recruitment and promote phenotype switch toward M2 macrophages during RIAKI. We also proved that EPO directly suppressed pro-inflammatory responses of M1 macrophages and promoted M2 marker expression in vitro. These results improve the understanding of the immunomodulatory capacity of EPO on macrophages, especially in the setting of $\mathrm{AKI}$, which might optimize the therapeutic modalities of EPO for sterile kidney injuries. A deep insight into the mechanisms of the immunomodulation of EPO can also help develop novel EPO derivatives with immunoregulatory functions.

\section{Materials and Methods}

Animals and rhabdomyolysis-induced AKI model. The Ethics Committee of Zhongshan hospital, Fudan University approved this study. All animal experiments were performed in accordance with established guidelines for the care and use of laboratory animals. C57/BL6J mice (male, 6-8 weeks old) were purchased from SLAC Laboratory (Shanghai, China), and housed in a pathogenfree, temperature-controlled environment with a 12-h light/dark cycle. Animals had free access to food and water. To induce rhabdomyolysis, mice were intramuscularly injected with 50\% glycerol (Sigma-Aldrich, Shanghai, China, $10 \mathrm{ml} / \mathrm{kg}$ ) into the hind limbs or saline as a control. To explore the possible protection of EPO against RIAKI, $500 \mathrm{IU} / \mathrm{kg}$ body weight of recombinant human EPO or PBS was administered intraperitoneally $30 \mathrm{~min}$ and $24 \mathrm{~h}$ after the glycerol injection. On day 2 after glycerol treatment, blood and kidney samples were collected for various detections.

Detection of kidney function. Serum creatinine and BUN, serving as indicators of kidney function, were measured using QuantiChrom Creatinine Assay Kit and QuantiChrom Urea Assay Kit (BioAssay Systems, Hayward, CA, USA) separately based on colorimetric assay according to the manufacturer's protocol.

Kidney histology. For histology, kidneys were fixed in $4 \%$ paraformaldehyde for $24 \mathrm{~h}$ and embedded in paraffin. Paraffin-embedded kidney blocks were cut into $2-\mu \mathrm{m}$ sections and then subjected to routine hematoxylin and eosin (H\&E) staining as well as periodic acid-Schiff (PAS) staining. Tissue sections were viewed by light microscope at $\times 200$ magnification. For semiquantitative analysis, five different fields at corticomedullary junction from each group were randomly selected. The histologic alterations in H\&E-stained slides were indicated as ATN score, which is graded on a scale of $0-5$ according to tubular necrosis, tubular dilation and cast formation (0, none; $1,<11 \% ; 2,11 \%$ to $25 \% ; 3,26 \%$ to $45 \% ; 4,46 \%$ to $75 \%$; $5,>75 \%$ ). The percentage area of PAS-stained brush border as well as tubular casts was quantified by using ImageJ software. Such histologic evaluations were performed in a blinded manner by experienced researchers.

TdT mediated dUTP nick end labeling (TUNEL) assay. To detect renal apoptosis in response to RIAKI, TUNEL staining was performed using In Situ Cell Death Detection Kit (Roche, Indianapolis, IN, USA) according to the manufacturer's instruction. The number of apoptotic cells was counted under light microscope at $\times 200$ magnification. At least 5 areas at the corticomedullary junction in the sections from different mice of each group were determined and averaged. 
Table 1 Primers used for qPCR analysis

\begin{tabular}{|c|c|c|}
\hline Genes & Forward primer $\left(5^{\prime}-3^{\prime}\right)$ & Reverse primer $\left(5^{\prime}-3^{\prime}\right)$ \\
\hline$K I M-1$ & TCCACACATGTACCAACATCAA & GTCACAGTGCCATTCCAGTC \\
\hline$N G A L$ & CCATCTATGAGCTACAAGAGAACAAT & TCTGATCCAGTAGCGACAGC \\
\hline$T N F-a$ & TCTTCTCATTCCTGCTTGTGG & GGTCTGGGCCATAGAACTGA \\
\hline$I L-1 \beta$ & TGTAATGAAAGACGGCACACC & TCTTCTTTGGGTATTGCTTGG \\
\hline$I L-6$ & GATGGATGCTACCAAACTGGA & CCAGGTAGCTATGGTACTCCAGAA \\
\hline IL-10 & ACCTGGTAGAAGTGATGCCCCAGGCA & CTATGCAGTTGATGAAGATGTCAAA \\
\hline$I L-12 b$ & AGGTCACACTGGACCAAAGG & AGGGTACTCCCAGCTGACCT \\
\hline$I L-17 A$ & TCCAGAAGGCCCTCAGACTA & CTCGACCCTGAAAGTGAAGG \\
\hline$I F N-\gamma$ & GAACTGGCAAAAGGATGGTGA & TGTGGGTTGTTGACCTCAAAC \\
\hline CCL2 & GTCCCTGTCATGCTTCTGG & GCTCTCCAGCCTACTCATTG \\
\hline$C C L 7$ & TCTCTCACTCTCTTTCTCCACC & GGGATCTTTTGTTTCTTGACATAGC \\
\hline Arginase-1 & GTGAAGAACCCACGGTCTGT & CTGGTTGTCAGGGGAGTGTT \\
\hline$Y m-1$ & TTCTTGTCACAGGTCTGG & TCCTTAGCCCAACTGGTATAG \\
\hline Fizz-1 & AGGAACTTCTTGCCAATCCA & CTGGGTTCTCCACCTCTTCA \\
\hline CD206 & CCTGTGCTCGAGAGGATATG & GCAGTCTGCATACCACTTGT \\
\hline$\beta$-actin & CCACGAGCGGTTCCGATG & GCCACAGGATTCCATACCCA \\
\hline
\end{tabular}

Flow cytometry. Intact kidney tissues were decapsulated, diced, and digested in collagenase type IV $(1 \mathrm{mg} / \mathrm{ml}$, Stemcell Technologies, Vancouver, British Columbia, Canada) for $30 \mathrm{~min}$ at $37^{\circ} \mathrm{C}$. Kidney tissue suspension was then passed through $40 \mu \mathrm{m}$ Falcon meshes followed by red cell lysis. Single cell suspension was washed 3 times with PBS and incubated with anti-mouse CD45-Brilliant Violet 510, CD11b-PE/Cy7, F4/80-Alexa Fluor 647 and CD206-PE (Biolegend, SanDiego, CA, USA) for $1 \mathrm{~h}$ at $4^{\circ} \mathrm{C}$ and detected on FACSCaliber Analyzer (BD Biosciences, San Jose, CA, USA). Data was processed using FlowJo software (Ashland, OR, USA).

Immunohistochemistry. For immunohistochemical staining of $F 4 / 80$, paraffin-embedded kidney sections were rehydrated through a series of graded ethanol and then incubated in $0.3 \%$ hydrogen peroxide to block the endogenous peroxidase activity. Specific primary antibodies were used for the detection of F4/80 (1:100, Abcam, Cambridge, MA, USA).

Cell culture and treatment. Human renal proximal tubular epithelial cell line (HK-2) was obtained from ATCC and maintained in DMEM/F12 medium containing $10 \%$ FBS. HK-2 cells were treated with $10 \mathrm{mg} / \mathrm{ml}$ myoglobin to mimic rhabdomyolysis in vitro. Different concentrations $(25,50$, and $100 \mathrm{IU} / \mathrm{ml})$ of EPO and Nec-1 $(10 \mu \mathrm{M})$ were also used. HK-2 cells maintained in normal medium were used as the control.

Macrophage cell line RAW 264.7 was purchased from ATCC and maintained in DMEM medium containing $10 \%$ FBS. Bone marrow cells from mice tibia and femur were incubated with $1000 \mathrm{U} / \mathrm{ml}$ GM-CSF (PeproTech) for 5 days to generate bone marrow-derived macrophages (BMDM). LPS (1 mg/ml, Sigma-Aldrich)+IFN- $\gamma$ $(20 \mathrm{ng} / \mathrm{ml}$, PeproTech, Southfield, MI, USA) or IL-4 $(20 \mathrm{ng} / \mathrm{ml}$, PeproTech) were used for M1 or M2 polarization in RAW 264.7 cells and BMDM, respectively.

Annexin V/PI assay. Cell death was analyzed using Annexin V-FITC/PI apoptosis detection kit (Vazyme Biotech, Nanjing, China). According to the manufacturer's protocol, cells were collected and resuspended in $100 \mu \mathrm{l}$ binding buffer mixed with $5 \mu \mathrm{l}$ Annexin V-FITC reagent and 5ul PI reagent. After incubation for $15 \mathrm{~min}$ at room temperature in the dark, another $400 \mu \mathrm{l}$ binding buffer was added, and cells were measured by flow cytometry (Beckman Coulter, Fullerton, $\mathrm{CA}$, USA). Data was analyzed with FlowJo software. Annexin $\mathrm{V}^{-} / \mathrm{PI}^{-}$represents live cells; Annexin $\mathrm{V}^{+} / \mathrm{PI}^{-}$represents early apoptotic cells; Annexin $\mathrm{V}^{-} / \mathrm{PI}^{+}$reflects necrosis; double positivity included both late apoptosis and necrosis.

ELISA and Griess reaction. Murine TNF- $\alpha$, IL-1 $\beta$, IL- 6 , and human CCL2, CCL7 ELISA kit were purchased from R\&D Systems (Boston, MA, USA). The levels of these cytokines and chemokines in serum or culture supernatants were measured according to the manufacturer's protocols. NO level in culture supernatants was detected by griess reagent (Sigma-Aldrich) according to the manufacturer's instruction.

Real-time quantitative PCR. Total RNA was extracted from kidney tissues and cultured cells using Trizol reagent according to the manufacturer's protocol. CDNA was reverse-transcribed using Reverse Transcription Kit (Takara, Dalian, China). RT-qPCR was performed using SYBR Green PCR mix (Roche) on an ABI
Prism 7500HT Sequence Detection System (Applied Biosystems, Paisley, UK). Gene expression levels were presented as fold exchange that was normalized to $\beta$ actin in control group. Primer sequences are listed in Table 1.

Western blotting. Total proteins from cultured cells were separated on SDSpolyacrylamide gels and transferred onto nitrocellulose membranes. The membranes were blocked in $5 \%$ nonfat milk for $1 \mathrm{~h}$ at room temperature, and then incubated overnight at $4{ }^{\circ} \mathrm{C}$ with primary antibodies against cleaved caspase-3, caspase-3, p-Jak2 tyr1007/1008, Jak2, p-p38, p38, PI3K, p-STAT3, STAT3, p-STAT6, STAT6, arginase-1, $\beta$-actin (Cell Signaling Technology, 1:1000), MLKL and p-MLKL ser358 (Abcam, 1:1000). Blots were subsequently washed and incubated with secondary antibodies for $1 \mathrm{~h}$ at room temperature. The immunoblots were visualized by chemiluminescence and quantified using ImageJ software.

Statistical analysis. All data are presented as means \pm S.D. Statistical analysis was performed using the Student's $t$-test (between two groups) and oneway ANOVA (among three or more groups) by SPSS 19.0 software (SPSS, Inc., Armonk, NY, USA). The Scheffe test was used for post hoc analysis. $P<0.05$ was recognized as statistically significant.

\section{Conflict of Interest}

The authors declare no conflict of interest.

Acknowledgements. This study was supported by National Natural Science Foundation of China (grants 81400752 to CY, 81370852 to MX, 81270832 to RR, 81270833, 81570674 to TZ)

1. Huerta-Alardin AL, Varon J, Marik PE. Bench-to-bedside review: Rhabdomyolysis - an overview for clinicians. Crit Care 2005; 9: 158-169.

2. Bosch X, Poch E, Grau JM. Rhabdomyolysis and acute kidney injury. N Engl J Med 2009; 361: $62-72$.

3. Bywaters EG, Beall D. Crush Injuries with Impairment of Renal Function. Br Med J 1941; 1: 427-432.

4. Vanholder R, Sever MS, Erek E, Lameire N. Rhabdomyolysis. J Am Soc Nephrol 2000; 11: 1553-1561.

5. Chatzizisis YS, Misirli G, Hatzitolios Al, Giannoglou GD. The syndrome of rhabdomyolysis: complications and treatment. Eur J Intern Med 2008; 19: 568-574.

6. Woodrow G, Brownjohn AM, Turney JH. The clinical and biochemical features of acute renal failure due to rhabdomyolysis. Ren Fail 1995; 17: 467-474.

7. de Meijer AR, Fikkers BG, de Keijzer MH, van Engelen BG, Drenth JP. Serum creatine kinase as predictor of clinical course in rhabdomyolysis: a 5-year intensive care survey. Intensive Care Med 2003; 29: 1121-1125.

8. Melli G, Chaudhry V, Cornblath DR. Rhabdomyolysis: an evaluation of 475 hospitalized patients. Medicine (Baltimore) 2005; 84: 377-385.

9. Zhang L, Fu P, Wang L, Cai G, Zhang L, Chen D et al. The clinical features and outcome of crush patients with acute kidney injury after the Wenchuan earthquake: differences between elderly and younger adults. Injury 2012; 43: 1470-1475.

10. Heyman SN, Rosen S, Rosenberger C. Animal models of renal dysfunction: acute kidney injury. Expert Opin Drug Discov 2009; 4: 629-641. 
11. Paller MS. Hemoglobin- and myoglobin-induced acute renal failure in rats: role of iron in nephrotoxicity. Am J Physiol 1988; 255: F539-F544.

12. Zager RA, Gamelin LM. Pathogenetic mechanisms in experimental hemoglobinuric acute renal failure. Am J Physiol 1989; 256: F446-F455.

13. Zager RA. Combined mannitol and deferoxamine therapy for myohemoglobinuric renal injury and oxidant tubular stress. Mechanistic and therapeutic implications. J Clin Invest 1992; 90: 711-719.

14. Kim JH, Lee SS, Jung MH, Yeo HD, Kim HJ, Yang Jl et al. N-acetylcysteine attenuates glycerol-induced acute kidney injury by regulating MAPKs and Bcl-2 family proteins. Nephrol Dial Transplant 2010; 25: 1435-1443.

15. Fahling M, Mathia S, Paliege A, Koesters R, Mrowka R, Peters $\mathrm{H}$ et al. Tubular von HippelLindau knockout protects against rhabdomyolysis-induced AKI. J Am Soc Nephrol 2013; 24: 1806-1819.

16. Zarjou A, Bolisetty S, Joseph R, Traylor A, Apostolov EO, Arosio P et al. Proximal tubule $\mathrm{H}$-ferritin mediates iron trafficking in acute kidney injury. J Clin Invest 2013; 123: 4423-4434.

17. Homsi E, Ribeiro-Alves MA, Lopes de Faria JB, Dias EP. Interleukin-6 stimulates tubular regeneration in rats with glycerol-induced acute renal failure. Nephron 2002; 92: 192-199.

18. Homsi E, Janino P, de Faria JB. Role of caspases on cell death, inflammation, and cell cycle in glycerol-induced acute renal failure. Kidney Int 2006; 69: 1385-1392.

19. Homsi E, Janino P, Amano M, Saraiva Camara NO. Endogenous hepatocyte growth factor attenuates inflammatory response in glycerol-induced acute kidney injury. Am J Nephrol 2009; 29: 283-291.

20. Geng Y, Zhang L, Fu B, Zhang J, Hong Q, Hu J et al. Mesenchymal stem cells ameliorate rhabdomyolysis-induced acute kidney injury via the activation of M2 macrophages. Stem Cell Res Ther 2014; 5: 80.

21. Belliere J, Casemayou A, Ducasse L, Zakaroff-Girard A, Martins F, lacovoni JS et al. Specific macrophage subtypes influence the progression of rhabdomyolysis-induced kidney injury. J Am Soc Nephrol 2015; 26: 1363-1377.

22. Komada T, Usui F, Kawashima A, Kimura H, Karasawa T, Inoue $Y$ et al. Role of NLRP3 Inflammasomes for Rhabdomyolysis-induced Acute Kidney Injury. Sci Rep 2015; 5: 10901.

23. Nishida K, Watanabe H, Ogaki S, Kodama A, Tanaka R, Imafuku T et al. Renoprotective effect of long acting thioredoxin by modulating oxidative stress and macrophage migration inhibitory factor against rhabdomyolysis-associated acute kidney injury. Sci Rep 2015; 5: 14471.

24. Maiese K, Li F, Chong ZZ. New avenues of exploration for erythropoietin. JAMA 2005; 293: 90-95.

25. Ghezzi P, Bernaudin M, Bianchi R, Blomgren K, Brines M, Campana W et al. Erythropoietin: not just about erythropoiesis. Lancet 2010; 375: 2142.

26. Nairz M, Schroll A, Moschen AR, Sonnweber T, Theurl M, Theurl I et al. Erythropoietin contrastingly affects bacterial infection and experimental colitis by inhibiting nuclear factor-kappaB-inducible immune pathways. Immunity 2011; 34: 61-74.

27. Cravedi P, Manrique J, Hanlon KE, Reid-Adam J, Brody J, Prathuangsuk P et al. Immunosuppressive effects of erythropoietin on human alloreactive T cells. J Am Soc Nephrol 2014; 25: 2003-2015.

28. Wiedenmann T, Ehrhardt S, Cerny D, Hildebrand D, Klein S, Heeg K et al. Erythropoietin acts as an anti-inflammatory signal on murine mast cells. Mol Immunol 2015; 65: 68-76.

29. Luo B, Gan W, Liu Z, Shen Z, Wang J, Shi R et al. Erythropoeitin Signaling in Macrophages Promotes Dying Cell Clearance and Immune Tolerance. Immunity 2016; 44: 287-302.

30. Luo B, Wang J, Liu Z, Shen Z, Shi R, Liu YQ et al. Phagocyte respiratory burst activates macrophage erythropoietin signalling to promote acute inflammation resolution. Nat Commun 2016; 7: 12177

31. Linkermann A, Green DR. Necroptosis. N Engl J Med 2014; 370: 455-465.

32. Linkermann A, Chen G, Dong G, Kunzendorf U, Krautwald S, Dong Z. Regulated cell death in AKI. J Am Soc Nephrol 2014; 25: 2689-2701.

33. Wang S, Zhang C, Hu L, Yang C. Necroptosis in acute kidney injury: a shedding light. Cell Death Dis 2016; 7: e2125.

34. Wang Z, Shah SV, Liu H, Baliga R. Inhibition of cytochrome P450 2E1 and activation of transcription factor Nrf2 are renoprotective in myoglobinuric acute kidney injury. Kidney Int 2014; 86: 338-349.

35. He S, Huang S, Shen Z. Biomarkers for the detection of necroptosis. Cell Mol Life Sci 2016; 73: $2177-2181$

36. Griffith JW, Sokol CL, Luster AD. Chemokines and chemokine receptors: positioning cells for host defense and immunity. Annu Rev Immunol 2014; 32: 659-702.

37. Luo B, Jiang M, Yang X, Zhang Z, Xiong J, Schluesener HJ et al. Erythropoietin is a hypoxia inducible factor-induced protective molecule in experimental autoimmune neuritis. Biochim Biophys Acta 2013; 1832: 1260-1270.

38. Lee TS, Lu KY, Yu YB, Lee HT. Beta common receptor mediates erythropoietin-conferred protection on OxLDL-induced lipid accumulation and inflammation in macrophages. Mediators of Inflammation 2015; 2015: 1-13.

39. Wood MA, Goldman N, DePierri K, Somerville J, Riggs JE. Erythropoietin increases macrophage-mediated T cell suppression. Nat Commun 2016; 306-307: 17-24.

40. Alnaeeli M, Raaka BM, Gavrilova O, Teng R, Chanturiya T, Noguchi CT. Erythropoietin signaling: a novel regulator of white adipose tissue inflammation during diet-induced obesity. Diabetes 2014; 63: 2415-2431.
41. Joshi D, Abraham D, Shiwen X, Baker D, Tsui J. Potential role of erythropoietin receptors and ligands in attenuating apoptosis and inflammation in critical limb ischemia. J Vasc Surg 2014; 60: 191-201 201.e1-2.

42. Liu Y, Luo B, Han F, Li X, Xiong J, Jiang M et al. Erythropoietin-derived nonerythropoietic peptide ameliorates experimental autoimmune neuritis by inflammation suppression and tissue protection. PLOS ONE 2014; 9: e90942.

43. Liu Y, Luo B, Shi R, Wang J, Liu Z, Liu W et al. Nonerythropoietic Erythropoietin-derived peptide suppresses adipogenesis, inflammation, obesity and insulin resistance. Sci Rep 2015; 5: 15134.

44. Watanabe M, Lundgren $T$, Saito $Y$, Cerami A, Brines M, Ostenson CG et al. A nonhematopoietic erythropoietin analogue, ARA 290, inhibits macrophage activation and prevents damage to transplanted islets. Transplantation 2016; 100: 554-562.

45. Lifshitz L, Tabak G, Gassmann M, Mittelman M, Neumann D. Macrophages as novel target cells for erythropoietin. Haematologica 2010; 95: 1823-1831.

46. Mantovani A, Sica A, Sozzani S, Allavena P, Vecchi A, Locati M. The chemokine system in diverse forms of macrophage activation and polarization. Trends Immunol 2004; 25: 677-686.

47. Mosser DM, Edwards JP. Exploring the full spectrum of macrophage activation. Nat Rev Immunol 2008; 8: 958-969.

48. Rees AJ. Monocyte and macrophage biology: an overview. Semin Nephrol2010; 30: 216-233.

49. Sica A, Mantovani A. Macrophage plasticity and polarization: in vivo veritas. J Clin Invest 2012; 122: 787-795.

50. Duffield JS. Macrophages and immunologic inflammation of the kidney. Semin Nephrol 2010; 30: 234-254.

51. Kawakami T, Lichtnekert J, Thompson LJ, Karna P, Bouabe H, Hohl TM et al. Resident renal mononuclear phagocytes comprise five discrete populations with distinct phenotypes and functions. J Immunol 2013; 191: 3358-3372.

52. Stamatiades EG, Tremblay ME, Bohm M, Crozet L, Bisht K, Kao D et al. Immune monitoring of trans-endothelial transport by kidney-resident macrophages. Cell 2016; 166 : 991-1003.

53. Jang HR, Rabb H. Immune cells in experimental acute kidney injury. Nat Rev Nephrol 2015; 11: 88-101.

54. Williams TM, Little MH, Ricardo SD. Macrophages in renal development, injury, and repair Semin Nephrol 2010; 30: 255-267.

55. Alikhan MA, Jones CV, Williams TM, Beckhouse AG, Fletcher AL, Kett MM et al. Colonystimulating factor-1 promotes kidney growth and repair via alteration of macrophage responses. Am J Pathol 2011; 179: 1243-1256.

56. Zhang MZ, Yao B, Yang S, Jiang L, Wang S, Fan X et al. CSF-1 signaling mediates recovery from acute kidney injury. J Clin Invest 2012; 122: 4519-4532.

57. Huen SC, Huynh L, Marlier A, Lee Y, Moeckel GW, Cantley LG. GM-CSF Promotes Macrophage Alternative Activation after Renal Ischemia/Reperfusion Injury. J Am Soc Nephrol 2015; 26: 1334-1345.

58. Wang Y, Chang J, Yao B, Niu A, Kelly E, Breeggemann MC et al. Proximal tubule-derived colony stimulating factor-1 mediates polarization of renal macrophages and dendritic cells, and recovery in acute kidney injury. Kidney Int 2015; 88: 1274-1282.

59. Chiba T, Skrypnyk NI, Skvarca LB, Penchev R, Zhang KX, Rochon ER et al. Retinoic Acid Signaling Coordinates Macrophage-Dependent Injury and Repair after AKI. J Am Soc Nephrol 2016; 27: 495-508.

60. Wang Y, Wang YP, Zheng G, Lee VW, Ouyang L, Chang DH et al. Ex vivo programmed macrophages ameliorate experimental chronic inflammatory renal disease. Kidney Int 2007; 72: 290-299.

61. Ko GJ, Boo CS, Jo SK, Cho WY, Kim HK. Macrophages contribute to the development of renal fibrosis following ischaemia/reperfusion-induced acute kidney injury. Nephrol Dial Transplant 2008; 23: 842-852.

62. Vernon MA, Mylonas KJ, Hughes J. Macrophages and renal fibrosis. Semin Nephrol 2010; 30: 302-317.

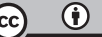

Cell Death and Disease is an open-access journal published by Nature Publishing Group. This work is licensed under a Creative Commons Attribution 4.0 International License. The images or other third party material in this article are included in the article's Creative Commons license, unless indicated otherwise in the credit line; if the material is not included under the Creative Commons license, users will need to obtain permission from the license holder to reproduce the material. To view a copy of this license, visit http://creativecommons.org/licenses/by/4.0/

C The Author(s) 2017 